\title{
Constructive Decision Theory
}

\author{
Lawrence Blume $^{a, c}$, David Easley ${ }^{a}$ and Joseph Y. Halpern ${ }^{b}$ \\ ${ }^{a}$ Dept. of Economics and Dept. of Information Science, Cornell University \\ ${ }^{b}$ Dept. of Computer Science, Cornell University \\ ${ }^{c}$ Institute for Advanced Studies, Vienna
}

July 5, 2021

\begin{abstract}
Supported in part by NSF under grants CTC-0208535, ITR-0325453, IIS-0534064, IIS1703846, and IIS-1718108, by ONR under grants N00014-00-1-03-41 and N00014-01-10511, by ARO under grant W911NF-17-1-0592, by a USDoD Multidisciplinary University Research Initiative (MURI) program administered by the ONR under grant N00014-01-1-0795, and by a MURI program administered by the ARO under grant W911NF-19-1-0217. An extended abstract of this paper (Blume et al., 2006) appeared in the Tenth International Conference on Principles of Knowledge Representation and Reasoning. An early version of this paper was written for and first presented at a conference on decision theory organized by Karl Vind in May 2004, shortly before his death. As a scholar and a colleague, Karl has left us all much to be grateful for. We are particularly grateful to him for pushing us to deliver a paper for this conference, and then to generously comment on it despite his ill health. We are also grateful to several individuals for comments, to the associate editor and reviewers of the paper, and to the many seminar audiences who have listened to and commented on this paper. In particular, we thank Jin-Yi Cai for suggesting the proof of Proposition_,
\end{abstract}


Abstract: In most contemporary approaches to decision making under uncertainty, a decision problem is described by a set of states and set of outcomes, and a rich set of acts, which are functions from states to outcomes over which the decision maker (DM) has preferences. Many interesting decision problems, however, do not come with a state space and an outcome space. Indeed, in complex problems it is often far from clear what the state and outcome spaces would be. We present an alternative foundation for decision making, in which the primitive objects of choice are syntactic programs. A representation theorem is proved in the spirit of standard representation theorems, showing that if the DM's preference relation on objects of choice satisfies appropriate axioms, then there exist a set $S$ of states, a set $O$ of outcomes, a way of interpreting the objects of choice as functions from $S$ to $O$, a probability on $S$, and a utility function on $O$, such that the DM prefers choice $a$ to choice $b$ if and only if the expected utility of $a$ is higher than that of $b$. Thus, the state space and outcome space are subjective, just like the probability and utility; they are not part of the description of the problem. In principle, a modeler can test for SEU behavior without having access to states or outcomes. We illustrate the power of our approach by showing that it can capture decision makers who are subject to framing effects and those who are subject to failures of extensionality.

\section{Correspondent:}

Professor David Easley

Department of Economics

Uris Hall

Cornell University

Ithaca NY 14850 
In memoriam Karl Vind.

\section{Introduction}

Models of decision making under uncertainty typically begin with states of the world, outcomes, acts, which are functions mapping states to outcomes, and preferences

over acts. This Savage (1954) presentation is convenient for the analysis of choice behavior, in particular for understanding how choices vary with those things the analyst interprets as variations in preferences such as tastes and beliefs. But this representation is often not close to the way in which choice problems appear in the world, and how decision makers (DMs) talk and reason about their decisions.

Decision problems are expressed not in the language of states, acts, and orders, but instead in some natural language in which the basic objects are statements such as 'the broker recommends a purchase of IBM' or 'the broker recommends a purchase of Alphabet' and the objects of choice are 'use my cash to buy IBM', 'use my cash to buy Alphabet', or 'do nothing'. The correct mapping of these statements into states and outcomes may seem obvious to the analyst, but what guarantees that the decision maker acts as if he uses any such mapping? The move from the language of decisions in the world to the language of decisions in theory is made by the analyst; it is subjective, it usually proceeds without discussion, and yet it determines the counterfactual claims that empirical economics uses to make causal assertions. Furthermore, much of the thrust of behavioral decision theory alludes to or even requires some unpacking of the DM's reasoning. This is difficult to do when the language of the model is so different from the language of the DM. In fact, Grabiszewski (2016) shows that the existence of an SEU representation for preferences in a decision problem has little empirical content. If the preference relation in a finite Savage presentation of a decision problem is complete, transitive, and satisfies a monotonicity requirement, then there exists some Savage representation into which the problem can be embedded in a preference-preserving way, and which has an SEU representation. This is simply to say that the empirical content of SEU preferences derives from the meaning of the states and outcomes. Starting with a natural-language description of a decision problem, we derive states, outcomes, and Savage acts in terms of the choices a DM can make and the things she can observe about the world, so that the Savage presentation is in some sense close to the DM's understanding of the problem. 
Our approach is to model choice objects as syntactic statements of the form 'if $t_{1}$ then choose $a_{1}$, else if $t_{2}$ choose $a_{2}, \ldots$ ', which we view as programs in a programming languge. The $a_{i}$ are actions (not 'acts') available to the DM. We call the $t_{i}$ 'tests'; they are propositions about the world. The programming language we focus on in this paper is very simple-we use it just to illustrate our ideas. Critically, it includes tests (in the context of if ...then ... else statements). These tests involve syntactic descriptions of the events in the world, and allow us to distinguish events from (syntactic) descriptions of events. In particular, there can be two different descriptions that, intuitively, describe the same event from the point of view of the modeler but may describe different events from the point of view of the decision maker. We assume that a DM has a preference relation on these programs 1 and from this derives a Savage representation: states, outcomes, acts, a corresponding preference relation on acts, and an expected utility representation for these preferences.

While bringing the description of choice problems closer to the way they appear in the world to DMs is a natural move in its own right, it also has several concrete advantages. First, empirical decision theory provides joint tests of the expected utility hypothesis and the analyst's interpretation as a Savage model. It will become clear that the Savage representation of any nontrivial decision problem is not unique, that many different Savage representations could be deployed. Separating the DM's understanding of the problem from the analyst's interpretation has obvious advantages, among them the ability to determine which properties of choice are common to all Savage representations and which are representation-specific. This is particularly important for research that proposes to 'test rationality'. It would be unfortunate if rationality meant 'agreement with the experimenter's view of the world'. We prefer to create a more flexible framework for rational choice and then add rejectable axioms to generate sharper predictions.

Second, in our approach, framing anomalies can often be understood as a conflict between the modeler's and the DM's representations. Although we do not pursue it here, our approach allows us to model the effects of changing the DM's view of the world by considering more (or a different set of) tests. Like Tversky and Koehler (1994), but unlike Ahn and Ergin (2007), our approach to framing begins with a natural-language description of the world. Unlike Tversky and Koehler, we construct a state space from the natural-language tests rather than taking it as externally given.

\footnotetext{
${ }^{1}$ Or, more precisely, as a referee pointed out, on the programs to which these syntactic descriptions refer.
} 
Third, our approach provides a natural way to capture resource-bounded reasoning - and even incorrect reasoning - about the world in an expected-utility framework. Fourth, our framework can model the interaction of DMs who describe the world in different ways. Finally, the modelling of many behavioral effects has often required the application of decision theories that are somewhat difficult to manipulate, for instance, requiring non-additive beliefs, menu-choice constructions, and the like. These models are often difficult to compare with each other, and they can be very difficult to implement. The basic expected utility framework is durable because although it is not great, it is often not bad; and because additive separability makes for representations that are easy to implement empirically. Our approach extends the domain of additive separability some way into regimes that have normatively been labelled irrational. The natural-language modeling approach provides additional hooks for theorizing about anomalous behavior within an expected-utility framework.

We began this project because we believed that for many decision problems, the assumption that there is a natural state space that would describe the uncertainty, and that DMs could (implicitly) articulate this in their reasoning, is ridiculous. Equally absurd is the assumption that in such circumstances individuals could articulate a complete preference relation over all alternatives. We therefore choose to allow for incomplete preferences. Of course, with incompleteness comes a loss of uniqueness of the representation. This does not concern us because, as we have observed, the the way the DM models the original natural-language problem in terms of states and outcomes is not unique either. On the other hand, we show that there always is a canonical representation, and the description of a state will be different depending on whether preferences are incomplete or not, and if so, what is missing.

Many papers in the literature raise issues with the state-space approach of Savage, or derive a subjective state space. Machina (2006) surveys the standard approach and illustrates many difficulties with the theory and with its uses. These difficulties include the ubiquitous ambiguity over whether the theory is meant to be descriptive or normative, whether states are exogenous or constructed by the DM, whether states are external to the DM, and whether they are measurable or not. Kreps (1992) and Dekel et al. (2001) use a menu choice model to deal with unforeseen contingencies - an inability of the DM to list all possible states of the world. They derive a subjective state space that represents possible preference relations over elements of the menu chosen by the DM. Ghirardato (2001) takes an alternative approach to unforeseen contingencies and models acts as correspondences from a state space to outcomes. Gilboa and Schmeidler (2004) and Karni (2006) raise 
objections to the state space that are similar to ours, and develop decision theories without a state space. Both papers derive subjective probabilities directly on outcomes. Ahn (2008) also develops a theory without a state space; in his theory, the DM chooses over sets of lotteries over consequences. Ahn and Ergin (2007) allow for the possibility that there may be different descriptions of a particular event, and use this possibility to capture framing. For them, a 'description' is a partition of the state space. They provide an axiomatic foundation for decision making in this framework, built on Tversky and Koehler's (1994) notion of support theory. (As we shall see, our approach is also quite compatible with support theory.) Grabiszewski (2016) asks whether a decision maker's preferences over acts mapping a given state space to a known outcome space can be SEU-rationalized with an alternative state space even if the original preferences were inconsistent with SEU. His analysis is related to our analysis in Section 4.3 with an objective outcome space. The primary difference is that we do not begin with states and outcomes; rather our DM has preferences over objects of choice (programs) described in his own language. Billot and Vergopoulos (2018) fix a state space and outcome space, but allow the DM to have a subjective interpretation of what they call feasible acts, which can be viewed as syntactic objects; each feasible act is interpreted as a function from states to outcomes. Finally, Lipman (1999) also considers language and uses a subjective state space that, intuitively, may include 'impossible possible worlds', where the standard axioms of logic may not hold. He shows how his approach can capture framing problems, among other things. Although there is clearly some overlap in intuitions, the technical details of our approach are significantly different from those mentioned above. Perhaps the closest is the work of Lipman; we discuss its relation to our work in Section 3.3.

The rest of this paper is organized as follows. In the next section, we introduce the syntactic programs that we take as our objects of choice, discuss several interpretations of the model, and show how syntactic programs can be interpreted as Savage acts. This section also includes several examples illustrating the power of our approach. In Section 3, we present our assumptions on preferences. Because the set of programs does not have a mixture-space structure, we replace independence assumptions with Krantz et al.'s (1971) cancellation axiom. In Section 4 we present our representation theorems for decision problems with subjective outcomes and those with objective outcomes. Section 5 discusses how our framework can model boundedly rational reasoning. In Section 6 we discuss how updating works for new information about the external world as well as for new information about preferences. Our goal in this paper is to introduce our approach and to relate it to the classical Savage approach. We conclude in Section 7 with a discussion of the 
benefits of the approach and further suggestions for how it can be applied.

\section{Describing Decision Problems}

As usual, we assume that the agent chooses among acts, but as we said in the introduction, for us, the acts are programs in a simple programming language. So we begin by describing the language of tests, and then use this language to construct programs, our syntactic objects of choice. We then discuss how the language of tests is used to describe the DMs theory of the world. We conclude the section by giving a several examples where this approach can capture behavior that is difficult to explain using more standard approaches.

\subsection{Languages for tests and choices}

A primitive test is a statement about the world that is either true or false, such as 'the economy will be strong next year' and 'the moon is in the seventh house'. We assume a finite set $T_{0}$ of primitive tests. The set $T$ of tests is constructed by closing the set of primitive tests under conjunction and negation. That is, $T$ is the smallest set such that $T_{0} \subseteq T$, and if $t_{1}$ and $t_{2}$ are in $T$, so is $t_{1} \wedge t_{2}$ and $\neg t_{1}$. Thus, the language of tests is just a propositional language whose atomic propositions are the elements of $T_{0}$.

We consider two languages for choices. In both cases, we begin with a finite set $\mathcal{A}_{0}$ of primitive choices. These may be objects such as 'buy 100 shares of IBM' or 'buy $\$ 10,000$ worth of bonds'. The interpretation of these acts is tightly bound to the decision problem being modeled. The first language simply closes off $\mathcal{A}_{0}$ under if ... then ... else. By this we mean that if $t$ is a test in $T$ and $a$ and $b$ are choices in $\mathcal{A}$, then if $t$ then $a$ else $b$ is also a choice in $\mathcal{A}$. When we need to be clear about which $T_{0}$ and $A_{0}$ were used to construct $\mathcal{A}$, we will write $\mathcal{A}_{\mathcal{A}_{0}, T_{0}}$. Note that $\mathcal{A}$ allows nesting, so that if $t_{1}$ then $a$ else (if $t_{2}$ then $b$ else $c$ ) is also a choice.

The second languages closes off $\mathcal{A}_{0}$ with if ... then ... else and randomization. That is, we assume that objective probabilities are available, and require that for any $0 \leq r \leq 1$, if $a$ and $b$ are choices, so is $r a+(1-r) b$. Randomization and if 
... then ... else can be nested in arbitrary fashion. We call this language $\mathcal{A}^{+}\left(\mathcal{A}_{\mathcal{A}_{0}, T_{0}}^{+}\right.$ when necessary).

Tests in $T$ are elements of discourse about the world. They could be events upon which choice is contingent: If the noon price of stock today is below $\$ 600$, then buy 100 shares, else buy none. More generally, tests in $T$ are part of the DM's description of the decision problem, just as states are part of the description of the decision problem in Savage's framework. However, elements of $T$ need not be complete descriptions of the relevant world, and therefore may not correspond to Savage's states. When we construct state spaces, elements of $T$ will clearly play a role in defining states, but, for some of our representation theorems, states cannot be constructed out of elements of $T$ alone. Additional information in states is needed for both incompleteness of preferences and when the outcome space is taken to be objective or exogenously given.

The choices in $\mathcal{A}$ and $\mathcal{A}^{+}$are syntactic objects; strings of symbols. They can be given semantics - that is, they can be interpreted-in a number of ways. For most of this paper we focus on one particular way of interpreting them that lets us connect them to Savage acts, but we believe that other semantic approaches will also prove useful (see Section [7). The first step in viewing choices as Savage acts is to construct a state space $S$, and to interpret the tests as events (subsets of $S$ ). With this semantics for tests, we can then construct, for the state space $S$ and a given outcome space $O$, a function $\rho_{S O}$ that associates with each choice $a$ a Savage act $\rho_{S O}(a)$, that is, a function from $S$ to $O$. Given a state space $S$, these constructions work as follows:

Definition 1. A test interpretation $\pi_{S}$ for the state space $S$ is a function associating with each test a subset of $S$. An interpretation is standard if it interprets $\neg$ and $\wedge$ in the usual way; that is

$$
\begin{aligned}
& \text { - } \pi_{S}\left(t_{1} \wedge t_{2}\right)=\pi_{S}\left(t_{1}\right) \cap \pi_{S}\left(t_{2}\right) \\
& \text { - } \pi_{S}(\neg t)=S-\pi_{S}(t) .
\end{aligned}
$$

Intuitively, $\pi_{S}(t)$ is the set of states where $t$ is true. Up to Section [5, we assume that all interpretations are standard, that is, the obey the rules of classical logic. In particular, this means that in all states, exactly one of $t$ or $\neg t$ is true. A standard interpretation is completely determined by its behavior on primitive tests. 
Definition 2. A choice interpretation $\rho_{S O}$ for the state space $S$ and outcome space $O$ assigns to each choice $a \in \mathcal{A}$ a (Savage) act, that is, a function $\rho_{S O}(a): S \rightarrow O$.

Given a test interpretation $\pi_{S}$ and a choice interpretation $\rho_{S O}^{0}: \mathcal{A}_{0} \rightarrow O^{S}$ for primitive choices, which assigns to each $a_{o} \in \mathcal{A}_{0}$ a function from $S \rightarrow O$, we can construct a choice interpretation by extending $\rho_{S O}^{0}$ inductively as follows:

$$
\rho_{S O}\left(\text { if } t \text { then } a_{1} \text { else } a_{2}\right)(s)= \begin{cases}\rho_{S O}\left(a_{1}\right)(s) & \text { if } s \in \pi_{S}(t) \\ \rho_{S O}\left(a_{2}\right)(s) & \text { if } s \notin \pi_{S}(t) .\end{cases}
$$

A choice interpretation $\rho_{S O}$ constructed in this way is said to be compatible with $\pi_{S}$ (and $\rho_{S O}^{0}$ ). This semantics captures the idea of contingent choices; that, in the choice if $t$ then $a_{1}$ else $a_{2}$, the realization of $a_{1}$ is contingent upon $t$, while $a_{2}$ is contingent upon 'not $t$ '. Of course, $a_{1}$ and $a_{2}$ could themselves be non-primitive programs, with nested if ... then ... else statements.

Extending the semantics to the language $\mathcal{A}^{+}$, given $S, O$, and $\pi_{S}$, requires us to associate with each choice $a$ an Anscombe-Aumann (AA) act (Anscombe and Aumann, 1963), that is, a function from $S$ to probability measures on $O$. Let $\Delta(O)$ denote the set of probability measures on $O$ and let $\Delta^{*}(O)$ be the subset of $\Delta(O)$ consisting of the probability measures that put probability one on an outcome. Let $\rho_{S O}^{0}: \mathcal{A}_{0} \rightarrow \Delta^{*}(O)^{S}$ be a choice interpretation for primitive choices that assigns to each $a_{o} \in \mathcal{A}_{0}$ a function from $S \rightarrow \Delta^{*}(O)$. Now we can extend $\rho_{S O}^{0}$ by induction on structure to all of $\mathcal{A}^{+}$in the obvious way. For if ... then ... else choices we use (1); to deal with randomization, define

$$
\rho_{S O}\left(r a_{1}+(1-r) a_{2}\right)(s)=r \rho_{S O}\left(a_{1}\right)(s)+(1-r) \rho_{S O}\left(a_{2}\right)(s) .
$$

That is, the distribution $\rho_{S O}\left(r a_{1}+(1-r) a_{2}\right)(s)$ is the obvious mixture of the distributions $\rho_{S O}\left(a_{1}\right)(s)$ and $\rho_{S O}\left(a_{2}\right)(s)$. Note that we require $\rho_{S O}$ to associate with each primitive choice in each state a single outcome (technically, a distribution that assigns probability 1 to a single outcome), rather than an arbitrary distribution over outcomes. So primitive choices are interpreted as Savage acts, and more general choices, which are formed by taking objective mixtures of choices, are interpreted as AA acts. This choice is largely a matter of taste. We would get similar representation theorems even if we allowed $\rho_{S O}^{0}$ to be an arbitrary function from $\mathcal{A}$ to $\Delta(O)^{S}$. However, this choice does matter for our interpretation of the results; see Example 10 for further discussion of this issue. 


\subsection{The DM's Theory of the World}

The DM will typically have some knowledge about relationships between various tests. For example, a DM that can do propositional reasoning will realize that $t_{1} \wedge t_{2}$ is equivalent to $t_{2} \wedge t_{1}$. A DM may also have domain-dependent knowledge. For example, if the DM knows that interest rates will remain constant between periods 2 and 3 , and interest rates are either $4 \%$ or $5 \%$, if $R_{i}(j)$ says that the interest rate in period $i$ is $j \%$, then the DM knows that

$$
\left(R_{2}(4) \vee R_{2}(5)\right) \wedge\left(R_{2}(4) \Leftrightarrow R_{3}(4)\right) \wedge\left(R_{2}(5) \Leftrightarrow R_{3}(5)\right) .
$$

Formally, we add to the description of a decision problem a theory, that is, a set $\mathcal{T} \subseteq T$ of tests.

Definition 3. A test interpretation $\pi_{S}$ for the state space $S$ respects a theory $\mathcal{T}$ iff for all $t \in \mathcal{T}, \pi_{S}(t)=S$.

A theory represents the DM's view of the world. Different people may, however, disagree about what they take to be obviously true of the world. Many people will assume that the sun will rise tomorrow. Others, like Laplace, will consider the possibility that it will not.

Choices $a$ and $b$ are equivalent with respect to a set $\Pi$ of test interpretations if, no matter what interpretation $\pi \in \Pi$ is used, they are interpreted as the same function. For example, in any standard interpretation, if $t$ then $a$ else $b$ is equivalent to if $\neg \neg t$ then $a$ else $b$; no matter what the test $t$ and choices $a$ and $b$ are, these two choices have the same input-output semantics.

Definition 4. For a set $\Pi$ of test interpretations, choices $a$ and $b$ are $\Pi$-equivalent, denoted $a \equiv_{\Pi} b$, if for all test interpretations $\pi \in \Pi$, if $\pi$ is a test interpretation on a state space $S$, then for all outcome spaces $O$ and choice interpretations $\rho_{S O}$ compatible with $\pi_{S}$, we have $\rho_{S O}(a)=\rho_{S O}(b)$.

Denote by $\Pi_{\mathcal{T}}$ the set of all standard interpretations that respect theory $\mathcal{T}$. Then $\Pi_{\mathcal{T}}$-equivalent $a$ programs and $b$ are said to be $\mathcal{T}$-equivalent, and we write $a \equiv_{\mathcal{T}} b$. Note that equivalence is defined relative to a given set $\Pi$ of interpretations. Two choices may be equivalent with respect to the set of all standard interpretations that 
hold a particular test $t$ to be true, but not equivalent to the larger set of all standard test interpretations.

In this section, we demonstrate the power of our approach by developing some well-known examples of framing. Framing problems appear when a DM solves inconsistently two decision problems that are designed by the modeler to be equivalent or that are obviously similar after recognizing an equivalence. The fact that choices are syntactic objects allows us to capture framing effects. This is an explicit virtue of providing a framework for decision theory that is closer to the natural language DMs might use in considering their choices.

Example 1. Consider the following well-known example of the effects of framing, due to McNeil et al. (1982). DMs are asked to choose between surgery or radiation therapy as a treatment for lung cancer. The problem is framed in two ways. In the survival frame, DMs are told that, of 100 people having surgery, 90 live through the post-operative period, 68 are alive at the end of the first year, and 34 are alive at the end of five years; and of 100 people have radiation therapy, all live through the treatment, 77 are alive at the end of the first year, and 22 are alive at the end of five years. In the mortality frame, DMs are told that of 100 people having surgery, 10 die during the post-operative period, 32 die by the end of the first year, and 66 die by the end of five years; and of 100 people having radiation therapy, none die during the treatment, 23 die by the end of the first year, and 78 die by the end of five years. Inspection shows that the outcomes are equivalent in the two frames-90 of 100 people living is the same as 10 out of 100 dying, and so on. Although one might have expected the two groups to respond to the data in similar fashion, this was not the case. While only $18 \%$ of DMs prefer radiation therapy in the survival frame, the number goes up to $44 \%$ in the mortality frame.

We can represent this example in our framework as follows. We assume that we have the following tests:

- $R T$, which intuitively represents '100 people have radiation therapy';

- $S$, which intuitively represents '100 people have surgery';

- $L_{i}(k)$, for $i=0,1,5$ and $k=0, \ldots, 100$, which intuitively represents that $k$ out of 100 people live through the post-operative period (if $i=0$ ), are alive after the first year (if $i=1$ ), and are alive after five years (if $i=5$ ); 
- $D_{i}(k)$, for $i=0,1,5$ and $k=0, \ldots, 100$, which is like $L_{i}(k)$, except 'live/alive' are replaced by 'die/dead'.

In addition, we assume that we have primitive programs $a_{S}$ and $a_{R}$ that represent 'perform surgery' and 'perform radiation theory'. With these tests, we can characterize the description of the survival frame by the following test $t_{1}$ :

$$
\left(S \Rightarrow L_{0}(90) \wedge L_{1}(68) \wedge L_{5}(34)\right) \wedge\left(R T \Rightarrow L_{0}(100) \wedge L_{1}(77) \wedge L_{5}(22)\right),
$$

(where, as usual, $t \Rightarrow t^{\prime}$ is an abbreviation for $\neg\left(t \wedge \neg t^{\prime}\right)$ ); similarly, the mortality frame is characterized by the following test $t_{2}$ :

$$
\left(S \Rightarrow D_{0}(10) \wedge D_{1}(32) \wedge D_{5}(66)\right) \wedge\left(R T \Rightarrow D_{0}(0) \wedge D_{1}(23) \wedge D_{5}(78)\right) .
$$

The choices offered in the McNeil et al. experiment can be viewed as conditional choices: what would a DM do conditional on $t_{1}$ (resp., $t_{2}$ ) being true. Using ideas from Savage, we can capture the survival frame as a decision problem with the following two choices:

$$
\begin{aligned}
& \text { if } t_{1} \text { then } a_{S} \text { else } a \text {, and } \\
& \text { if } t_{1} \text { then } a_{R} \text { else } a \text {, }
\end{aligned}
$$

where $a$ is an arbitrary choice. Intuitively, comparing these choices forces the DM to consider his preferences between $a_{S}$ and $a_{R}$ conditional on the test, since the outcome in these two choices is the same if the test does not hold. Similarly, the mortality frame amounts to a decision problem with the analogous choices with $t_{1}$ replaced by $t_{2}$.

There is nothing in our framework that forces a DM to identify the tests $t_{1}$ and $t_{2}$; the tests $L_{i}(k)$ and $D_{i}(100-k)$ a priori are completely independent, even if the problem statement suggests that they should be equivalent. Hence there is no reason for a DM to identify the choices if $t_{1}$ then $a_{S}$ else $a$ and if $t_{2}$ then $a_{S}$ else $a$. As a consequence, as we shall see, it is perfectly consistent with our axioms that a DM has the preferences if $t_{1}$ then $a_{S}$ else $a \succ$ if $t_{1}$ then $a_{R}$ else $a$ and if $t_{2}$ then $a_{R}$ else $a \succ$ if $t_{2}$ then $a_{S}$ else $a$. a sophisticated DM might understand that $L_{i}(k) \Leftrightarrow D_{i}(100-k)$, for $i=0,1,5$ and $k=1, \ldots, 100$.

We view it as a feature of our framework that it can capture this framing example for what we view as the right reason: the fact that DMs do not necessarily 
identify $L_{i}(k)$ and $D_{i}(100-k)$. Of course, a sophisticated DM might understand that $L_{i}(k) \Leftrightarrow D_{i}(100-k)$, for $i=0,1,5$ and $k=1, \ldots, 100$. Such a DM would include these tests in her theory $\mathcal{T}$. If these tests are in her theory, then she will make the same decision in both frames. More precisely, we have if $t_{1}$ then $a_{S}$ else $a \equiv_{\mathcal{T}}$ if $t_{2}$ then $a_{S}$ else $a$ and if $t_{1}$ then $a_{R}$ else $a \equiv_{\mathcal{T}}$ if $t_{2}$ then $a_{R}$ else $a$. The results of the McNeil et al. experiment can be interpreted in our language as a failure by some DMs to have a theory that makes tests stated in terms of mortality data or survival data semantically equivalent. A similar approach can be used to capture 'nudges' or 'naive diversification'.

Example 2. As discussed at length by Thaler and Sunstein (2009), what the default choice is can have a significant effect on the outcome of a decision problem. For example, whether the default in a company's $401 \mathrm{~K}$ plan is that employees are enrolled in the plan, with the possibility of opting out, or the default is that employees are not enrolled in the plan, but have the option of opting in, can make a huge difference to participation rates.

We model this in our framework using an approach similar to that used in Example 1. There are two frames. In the first, the default is participation and the active option is to opt out; in the second, the default is not participating, and the active option is opting in. From the agent's perspective, there are two basic actions:

- $a_{1}$ : do nothing (which means that the agent will get the default option);

- $a_{2}$ : choose the active option.

Let $t_{1}$ be the formula that describes the first frame: if the agent does nothing then he participates in the $401 \mathrm{~K}$; otherwise, he fills in paperwork and opts out. Let $t_{2}$ be the corresponding formula that describes the second frame. This means that the agent is comparing if $t_{1}$ then $a_{1}$ else $a$ and if $t_{1}$ then $a_{2}$ else $a$ in the first frame, where $a$ is some default action, and comparing if $t_{2}$ then $a_{1}$ else $a$ and if $t_{2}$ then $a_{2}$ else $a$. As it happens, if $t_{1}$ then $a_{1}$ else $a$ and if $t_{2}$ then $a_{2}$ else $a$ (resp., if $t_{1}$ then $a_{2}$ else $a$ and if $t_{2}$ then $a_{1}$ else $a$ ) lead to the same outcome in the actual domain. A DM who is resource-bounded, however, may not recognize this cross-frame equivalence and chooses $a_{1}$ in both frames.

Example 3. Benartzi and Thaler (2001) observed that if employees are offered $n$ choices in a defined contribution savings plan, many naively diversify, using the 
heuristic of putting $1 / n$ of their allocation into each of the $n$ options offered. This, to us, shows the effect of language on choices.

The survey that Benartzi and Thaler use to illustrate this point asks employees to allocate their retirement savings between two funds: $A$ and $B$. In one instance, fund $A$ is a stock fund and fund $B$ is a bond fund. In the second instance, fund $A$ is a stock fund and fund $B$ is a balanced fund putting one-half of its money in stocks and onehalf in bonds. Benartzi and Thaler find that a large portion of participants split their retirement savings equally between $A$ and $B$ in both instances. To test whether this results from a misunderstanding of stocks and bonds, Benartzi and Thaler run the same survey with the terms stock and bond replaced by the distribution on returns that would result from each choice. They get the same answer.

In our framework a simplified version of this survey can be captured by a decision problem with two primitive actions:

- $a_{1}$ : put $1 / 2$ of the money in fund $A$ and $1 / 2$ in fund $B$;

- $a_{2}$ : put all of the money in fund B;

and two tests that describe the contents of the funds:

- $t_{1}$ : the formula describing the first frame in which fund $A$ is all stock and fund $B$ is all bonds;

- $t_{2}$ : the formula describing the second frame in which fund $A$ is all stock and fund $B$ is one-half stock and one-half bonds.

The decision maker compares if $t_{1}$ then $a_{1}$ else $a$ and if $t_{1}$ then $a_{2}$ else $a$ where $a$ is some default action, and compares if $t_{2}$ then $a_{1}$ else $a$ and if $t_{2}$ then $a_{2}$ else $a$. In this setting if $t_{1}$ then $a_{1}$ else $a$ and if $t_{2}$ then $a_{2}$ else $a$ lead to the same outcome. Not having thought about it, a DM may not realize if $t_{1}$ then $a_{1}$ else $a$ and if $t_{2}$ then $a_{2}$ else $a$ lead to the same outcome; indeed, may even believe that if $t_{1}$ then $a_{1}$ else $a$ and if $t_{2}$ then $a_{1}$ else $a$ lead to the same outcome.

Our approach restricts a theory to being a set of tests (although we consider a generalization of this in Section [5. The programs if $t_{1}$ then $a_{1}$ else $a$ and 
if $t_{2}$ then $a_{2}$ else $a$ may be equivalent in the mind of the experimenter, and perhaps also in the mind of the subject. Our language of tests is not rich enough to capture how the DM interprets programs, but this is captured by the DM's preference relation. If the DM views two programs as equivalent, then he will be indifferent between them.

Example 4. In a famous experiment, Johnson et al. (1993) have a dramatic finding, tied to a decision problem: Subjects offered hypothetical health insurance were willing to pay a higher premium for policies covering hospitalization for any disease than they were for policies covering hospitalization for any reason at all, which would include both disease and accidents. Tversky and Koehler (1994) understand this as a failure of extensionality. They observe (p. 548) that '... probability judgments are attached not to events but to descriptions of events'. Extensionality is the property that different descriptions of the same event should be assigned the same probability. It fails in the Johnson et al. experiment because the event 'any disease or accident' is a strict subset of 'any reason at all'; extensionality would require the probability of 'any reason at all' to be the sum of 'any disease or accident' and 'a reason other than disease of accident'. Tversky and Koehler respond to this failure by introducing support theory as a way of putting likelihoods not on events but on their descriptions.

In our framework, extensionality fails when two descriptions of the same event are not perceived as such by the DM. Because for us states are subjective, representing the DM's point of view, so even if tests $t_{1}$ and $t_{2}$ are equivalent from the modeler's point of view, we may have $\pi_{S}\left(t_{1}\right) \neq \pi_{S}\left(t_{2}\right)$ for the DM's state space $S$ and test interpretation $\pi_{S}$. Thus, the DM may assign $t_{1}$ and $t_{2}$ different probabilities.

The DM's theory captures equivalence among descriptions from the point of view of the DM. For instance, suppose a DM is considering the purchase of an home insurance policy. One alternative might offer coverage up to the value of the home and its contents 'in case of damage'. Another might offer the same coverage for 'storm damage', 'fire damage', and 'other damage'. The relevant tests are $t_{1}$, damage of any kind, and $t_{2}$ through $t_{4}$ : storm, fire, and other damage, respectively. If $t_{1} \Leftrightarrow\left(t_{2} \vee t_{3} \vee t_{4}\right)$ is not part of her theory $\mathcal{T}$, then the DM's decision problem might be represented by a state space $S$ and a test interpretation $\pi_{S}$ such that $\pi_{S}\left(t_{1}\right) \neq$ $\pi_{S}\left(t_{2}\right) \cup \pi_{S}\left(t_{3}\right) \cup \pi_{S}\left(t_{4}\right)$. On the other hand, should $t_{1} \Leftrightarrow\left(t_{2} \vee t_{3} \vee t_{3}\right)$ be part of her theory $\mathcal{T}$, then for any state space $S$ and test interpretation $\pi_{S} \in \Pi_{\mathcal{T}}, \pi_{S}\left(t_{1}\right)=$ $\pi_{S}\left(t_{2}\right) \cup \pi_{S}\left(t_{3}\right) \cup \pi_{S}\left(t_{4}\right)$.

Support theory develops this idea in a very specific way. Two events $A$ and 
$B$ are exclusive (with respect to an interpretation $\pi_{S}$ ) if, in our terminology, $\pi_{S}(A) \cap$ $\pi_{S}(B)=\emptyset$. Support theory distinguishes two kinds of disjunctions. If $t_{1}$ and $t_{2}$ are exclusive, their explicit disjunction is $t_{1} \vee t_{2}$. So in the preceding paragraph, ' $t_{2}$ or $t_{3}$ or $t_{4}$ ', that is, ' $t_{2} \vee t_{3} \vee t_{4}$ ', is an explicit disjunction. On the other hand, ' $t_{1}$ ' is an implicit disjunction; it is in fact the disjunction of $t_{2}$ and $t_{3}$ but it is not an 'or' statement. Support theory assumes the existence of a ratio scale $s$ that measures the degree of support for an event. The key idea of support theory is expressed in Tversky and Koehler's (1994) equation 2:

$$
s\left(t_{1}\right) \leq s\left(t_{2} \vee t_{3} \vee t_{4}\right)=s\left(t_{2}\right)+s\left(t_{3}\right)+s\left(t_{4}\right),
$$

that is, the scale $s$ is additive over explicit disjunction, but subadditive over implicit disjunction. Effectively, Tversky and Koehler are assuming that if $t_{1}$ is an implicit disjunction of $t_{2}$ and $t_{3}$, then the agent realizes that $t_{2} \Rightarrow t_{1}$ and that $t_{3} \Rightarrow t_{1}$, but not that $t_{1} \Leftrightarrow\left(t_{2} \vee t_{3}\right)$; that is, the first two formulas are in his theory, while the third is not. Under these assumptions, (2) seems perfectly reasonable.

'Implicit subadditivity' can be captured in our framework simply by allowing $\pi_{S}\left(t_{1}\right) \supseteq \pi_{S}\left(t_{2}\right) \cup \pi_{S}\left(t_{3}\right) \cup \pi_{S}\left(t_{4}\right)$. Subsequent iterations of support theory propose that subadditivity may also apply to explicit disjunctions, that is, $s\left(t_{2} \vee t_{3} \vee t_{4}\right) \leq s\left(t_{2}\right)+$ $s\left(t_{3}\right)+s\left(t_{4}\right)$. In our approach, this stronger form of subadditivity (and, more generally, non-additivity) can be captured by considering nonstandard test interpretations; see Section 5 .

We are not taking a stand on the normative implications of extensionality failures. Our point is that extensionality and its failure is a product of the theory of the world $\mathcal{T}$ that a DM brings to the decision problem at hand (and how it compares to the experimenter's or modeler's view of the world). One can extend conventional expected utility to state spaces and act spaces that admit failures of extensionality. A failure of extensionality is not inconsistent with the existence of an SEU representation on a suitably constructed state space. Moreover, if we modelers take $t_{0} \Leftrightarrow t_{1}$ to be an axiom that describes the world, but our DM disagrees, then the probability of the set $\left(\pi_{S}\left(t_{0}\right) / \pi_{S}\left(t_{1}\right)\right) \cup\left(\pi_{S}\left(t_{1}\right) / \pi_{S}\left(t_{0}\right)\right)$ measures the degree of framing bias from the modeler's point of view. Furthermore, our framework offers a natural way of bringing support theory to decision problems; a DM's degree of support for a formula could be inferred from individuals' willingness to accept certain bets.

In many experiments (e.g., that of Johnson et al. (1993)), some subjects are exposed to a treatment where they see only implicit disjunctions, while others 
are exposed only to the basic events. Different subjects are exposed to different frames, and different frames come with different languages, so there is no cause for surprise that extensionality fails; options available in one treatment are not present in the other treatment. It is also interesting to ask what happens when language changes during the course of evaluation. We might imagine a sequential problem in which subjects are first asked to make a broad assessment of why a car failed, and then are asked for more explicit assessments about the source of the failure. The second-stage questions add possibilities to the DM's language. We could certainly model this in a probabilistic way, which would show no subadditivity, but we can also imagine alternatives that would be consistent with failures of extensionality. This, however, is a topic for another paper.

\section{The Axioms}

This section lays out our basic assumptions on preferences. If we want to get an SEU representation, then we must have an analogue of an independence axiom or the sure-thing principle. However, in their usual form, these axioms require a mixture space. The set of feasible programs is not a mixture space. A standard way of dealing with this problem (see, e.g., (Krantz et al., 1971)) is to use cancellation axioms. Since cancellation is not so well known among economists, we also illustrate the relationship between cancellation and more familiar preference properties.

\subsection{Preferences}

We assume that the DM has a preference relation $\succ$ on a subset $C$ of the set $\mathcal{A}$ (resp., $\mathcal{A}^{+}$) of non-randomized (resp., randomized) acts. 2 This preference relation has the usual interpretation of 'at least as good as'. We take $a \succ b$ to be an abbreviation for $a \succeq b$ and $b \succeq a$, even if $\succeq$ is not complete. We prove various representation theorems that depend upon the language, and upon whether outcomes are taken to be given or not. The engines of our analysis are various cancellation axioms, which are the subject of the next section. At some points in our analysis we consider complete preferences:

\footnotetext{
${ }^{2}$ We do not assume that this relation is necessarily complete or transitive; it is just a binary relation on $C \times C$.
} 
A1. The preference relation $\succeq$ is complete.

The completeness axiom has often been defended by the claim that 'people, in the end, make choices'. Nonetheless, from the outset of modern decision theory, completeness has been regarded as a problem. Savage (1954, Section 2.6) discusses the difficulties involved in distinguishing between indifference and incompleteness. $\mathrm{He}$ concludes by choosing to work with the relationship he describes with the symbol $\leqq$, later abbreviated as $\leq$, which he interprets as 'is not preferred to'. The justification of completeness for the 'is not preferred to' relationship is anti-symmetry of strict preference. Savage, Aumann (1962), Bewley (2002) and Mandler (2001) argue against completeness as a requirement of rationality. Eliaz and OK (2006) have argued that rational choice theory with incomplete preferences is consistent with preference reversals. In our view, incompleteness is an important expression of ambiguity in its plain meaning (rather than as a synonym for a non-additive representation of likelihood). There are many reasons why a comparison between two objects of choice may fail to be resolved: obscurity or indistinctness of their properties, lack of time for or excessive cost of computation, the incomplete enumeration of a choice set, and so forth. We recognize indecisiveness in ourselves and others, so it would seem strange not to allow for it in any theory of preferences that purports to describe tastes (as opposed to a theory which purports to characterize consistent choice).

We will focus our analysis on preferences that may be incomplete. To us, requiring completeness for preferences over the complex syntactic objects in $\mathcal{A}$ seems unnaturally restrictive. Our axioms thus yield representation theorems for incomplete preferences. Once we obtain states, outcomes, and acts, our theorems are similar to other representation theorems for incomplete preferences (e.g., (Dubra et al., 2004, Galaabaatar and Karni, 2013, Nau, 2006)), although our axioms differ as they are placed on preferences over different objects. While representation theorems with incomplete preferences are, by now, quite standard, we are able to use them to give some interesting insight into updating (see Section 6).

\subsection{Cancellation}

Although simple versions of the cancellation axiom have appeared in the literature (e.g., Scott (1964) and Krantz et al. (1971)), it is nonetheless not well known, and so before turning to our framework we briefly explore some of its implications in more 
familiar settings. Nonetheless, some of the results here are new; in particular, the results on cancellation for partial orders. These will be needed for proofs in the appendix.

Let $C$ denote a set of choices and $\succeq$ a preference relation on $C$. We use the following notation: Suppose $\left\langle a_{1}, \ldots, a_{n}\right\rangle$ and $\left\langle b_{1}, \ldots, b_{n}\right\rangle$ are sequences of elements of $C$. If for all $c \in C, \#\left\{j: a_{j}=c\right\}=\#\left\{j: b_{j}=c\right\}$, we write $\left\{\left\{a_{1}, \ldots a_{n}\right\}\right\}=$ $\left\{\left\{b_{1}, \ldots, b_{n}\right\}\right\}$. That is, the multisets formed by the two sequences are identical.

Definition 5 (Cancellation). The preference relation $\succeq$ on $C$ satisfies cancellation iff for all pairs of sequences $\left\langle a_{1}, \ldots, a_{n}\right\rangle$ and $\left\langle b_{1}, \ldots, b_{n}\right\rangle$ of elements of $C$ such that $\left\{\left\{a_{1}, \ldots, a_{n}\right\}\right\}=\left\{\left\{b_{1}, \ldots, b_{n}\right\}\right\}$, if $a_{i} \succeq b_{i}$ for $i \leq n-1$, then $b_{n} \succeq a_{n}$.

Roughly speaking, cancellation says that if two collections of choices are identical, then it is impossible to order the choices so as to prefer each choice in the first collection to the corresponding choice in the second collection. The following proposition shows that cancellation is equivalent to reflexivity and transitivity. Although Krantz et al. (1971, p. 251) and Fishburn (1987, p. 743) have observed that cancellation implies transitivity, this full characterization appears to be new.

Proposition 1. A preference relation $\succeq$ on a choice set $C$ satisfies cancellation iff

(a) $\succeq$ is reflexive, and

(b) $\succeq$ is transitive.

\section{All proofs are provided in the Appendix.}

We use two strengthenings of cancellation in our representation theorems for $\mathcal{A}$ and $\mathcal{A}^{+}$, respectively. The first, statewise cancellation, simply applies cancellation to a setting where the objects of choice are functions; in that case, we apply cancellation to each argument of the function. We first state the statewise cancellation to Savage acts, which we view as functions from a finite set $S$ of states to a finite set $O$ of outcomes. Let $C$ denote a set of Savage acts and suppose that $\succeq$ is a preference relation on $C$.

Definition 6 (Statewise Cancellation). The preference relation $\succeq$ on a set $C$ of Savage acts satisfies statewise cancellation iff for all pairs of sequences $\left\langle a_{1}, \ldots, a_{n}\right\rangle$ and $\left\langle b_{1}, \ldots, b_{n}\right\rangle$ of elements of $C$, if $\left\{\left\{a_{1}(s), \ldots, a_{n}(s)\right\}\right\}=\left\{\left\{b_{1}(s), \ldots, b_{n}(s)\right\}\right\}$ for all $s \in S$, and $a_{i} \succeq b_{i}$ for $i \leq n-1$, then $b_{n} \succeq a_{n}$. 
As written, statewise cancellation is really an infinite family of postulates, one for each $n$. As we now show, we actually only need cancellation to hold for finitely many values of $n$. Let $S C_{n}$ be the instance of cancellation that involves pairs of sequences of length $n$.

Proposition 2. If $\succeq$ is a preference relation on a finite set $C$, then there exists $N$ such that $\succeq$ satisfies $S C_{n}, n=1,2,3, \ldots$ iff $\succeq$ satisfies $S C_{n}$ for all $n \leq N$.

The argument used to prove Proposition 2 applies with essentially no change to all later variants of cancellation that we consider. We believe it should be possible to obtain a bound on the number of instances of the cancellation postulate needed in terms of the cardinality of the set $C$ of Savage acts, although we have not proved this.

Statewise cancellation is a powerful assumption because equality of the multisets is required only 'pointwise'. Any pair of sequences that satisfy the conditions of cancellation also satisfies the conditions of statewise cancellation, but the converse is not true. For instance, suppose that $S=\left\{s_{1}, s_{2}\right\}$, and we use $\left(o_{1}, o_{2}\right)$ to refer to an act with outcome $o_{i}$ in state $i, i=1,2$. Consider the two sequences of acts $\left\langle\left(o_{1}, o_{1}\right),\left(o_{2}, o_{2}\right)\right\rangle$ and $\left\langle\left(o_{1}, o_{2}\right),\left(o_{2}, o_{1}\right)\right\rangle$. These two sequences satisfy the conditions of statewise cancellation, but not that of cancellation.

In addition to the conditions in Proposition 1 statewise cancellation directly implies event independence, a condition at the heart of SEU representation theorems (and which can be used to derive the Sure Thing Principle). If $T \subseteq S$, let $a_{T} b$ be the Savage act that agrees with $a$ on $T$ and with $b$ on $S-T$; that is $a_{T} b(s)=a(s)$ if $s \in T$ and $a_{T} b(s)=b(s)$ if $s \notin T$. We say that $\succeq$ satisfies event independence iff for all acts $a, b, c$, and $c^{\prime}$ and subsets $T$ of the state space $S$, if $a_{T} c \succeq b_{T} c$, then $a_{T} c^{\prime} \succeq b_{T} c^{\prime}$.

Proposition 3. If $\succeq$ satisfies statewise cancellation, then $\succeq$ satisfies event independence.

Proposition 1 provides a characterization of cancellation for choices in terms of familiar properties of preferences. We do not have a similarly simple characterization of statewise cancellation. In particular, the following example shows that it is not equivalent to the combination of reflexivity and transitivity of $\succeq$ and event independence. 
Example 5. Suppose that $S=\left\{s_{1}, s_{2}\right\}, O=\left\{o_{1}, o_{2}, o_{3}\right\}$. There are nine possible acts. Suppose that $\succeq$ is the smallest reflexive, transitive relation such that

$$
\begin{gathered}
\left(o_{1}, o_{1}\right) \succ\left(o_{1}, o_{2}\right) \succ\left(o_{2}, o_{1}\right) \succ\left(o_{2}, o_{2}\right) \succ\left(o_{3}, o_{1}\right) \succ \\
\left(o_{1}, o_{3}\right) \succ\left(o_{2}, o_{3}\right) \succ\left(o_{3}, o_{2}\right) \succ\left(o_{3}, o_{3}\right),
\end{gathered}
$$

using the representation of acts described above. To see that $\succeq$ satisfies event independence, note that

- $\left(x, o_{1}\right) \succeq\left(x, o_{2}\right) \succeq\left(x, o_{3}\right)$ for $x \in\left\{o_{1}, o_{2}, o_{3}\right\}$;

- $\left(o_{1}, y\right) \succeq\left(o_{2}, y\right) \succeq\left(o_{3}, y\right)$ for $y \in\left\{o_{1}, o_{2}, o_{3}\right\}$.

However, statewise cancellation does not hold. Consider the sequences

$$
\left\langle\left(o_{1}, o_{2}\right),\left(o_{2}, o_{3}\right),\left(o_{3}, o_{1}\right)\right\rangle \text { and }\left\langle\left(o_{2}, o_{1}\right),\left(o_{3}, o_{2}\right),\left(o_{1}, o_{3}\right)\right\rangle \text {. }
$$

This pair of sequences clearly satisfies the hypothesis of statewise cancellation, that $\left(o_{1}, o_{2}\right) \succeq\left(o_{2}, o_{1}\right)$ and $\left(o_{2}, o_{3}\right) \succeq\left(o_{3}, o_{2}\right)$, but $\left(o_{1}, o_{3}\right) \succeq\left(o_{3}, o_{1}\right)$.

For our representation theorems for complete orders, statewise cancellation suffices. However, for partial orders, we need a version of cancellation that is equivalent to statewise cancellation in the presence of $\mathbf{A 1}$, but is in general stronger.

Definition 7 (Extended Statewise Cancellation). The preference relation $\succeq$ on a set $C$ of Savage acts satisfies extended statewise cancellation if and only if for all pairs of sequences $\left\langle a_{1}, \ldots, a_{n}\right\rangle$ and $\left\langle b_{1}, \ldots, b_{n}\right\rangle$ of elements of $C$ such that $\left\{\left\{a_{1}(s), \ldots, a_{n}(s)\right\}\right\}=\left\{\left\{b_{1}(s), \ldots, b_{n}(s)\right\}\right\}$ for all $s \in S$, if there exists some $k<n$ such that $a_{i} \succeq b_{i}$ for $i \leq k, a_{k+1}=\cdots=a_{n}$, and $b_{k+1}=\cdots=b_{n}$, then $b_{n} \succeq a_{n}$.

With extended statewise cancellation, we require that the last $n-k$ elements in each of the sequences are equal. The fact that $b_{n} \succeq a_{n}$ thus means that $b_{j} \succeq a_{j}$ for $j=k+1, \ldots, n$. Note that statewise cancellation is the special case of extended statewise calculation where $k=n-1$.

Proposition 4. In the presence of $\mathbf{A 1}$, extended statewise cancellation and statewise cancellation are equivalent. 
The extension of cancellation needed for $\mathcal{A}^{+}$is based on the same idea as extended statewise cancellation, but probabilities of objects rather than the incidences of objects are added up. Let $C$ denote a collection of elements from a finite-dimensional mixture space. Thus, $C$ can be viewed as a subspace of $\mathbf{R}^{\mathbf{n}}$ for some $n$, and each component of any $c \in C$ is a probability. We can then formally 'add' elements of $C$, adding elements of $\mathbf{R}^{\mathbf{n}}$ pointwise. (Note that the result of adding two elements in $C$ is no longer an element of $C$, and in fact is not even a mixture.)

Definition 8 (Extended Mixture Cancellation). The preference relation $\succeq$ on $C$ satisfies extended mixture cancellation iff for all pairs of sequences $\left\langle a_{1}, \ldots, a_{n}\right\rangle$ and $\left\langle b_{1}, \ldots, b_{n}\right\rangle$ of elements of $C$, such that $\sum_{i=1}^{n} a_{i}=\sum_{i=1}^{n} b_{i}$, if there exists some $k<n$ such that $a_{i} \succeq b_{i}$ for $i \leq k, a_{k+1}=\cdots=a_{n}$, and $b_{k+1}=\cdots=b_{n}$, then $b_{n} \succeq a_{n}$.

We can extend Proposition 1 to get a characterization theorem for preferences on mixture spaces by using an independence postulate. The preference relation $\succeq$ satisfies mixture independence if for all $a, b$, and $c$ in $C$, and all $r \in(0,1], a \succeq b$ iff $r a+(1-r) c \succeq r b+(1-r) c$. The preference relation $\succeq$ satisfies rational mixture independence if it satisfies mixture independence for all rational $r \in(0,1]$.

Theorem 1. A preference relation $\succeq$ on a finite-dimensional mixture space $C$ satisfies extended mixture cancellation iff $\succeq$ is reflexive, transitive, and satisfies rational mixture independence.

\subsection{The cancellation postulate for choices}

We use cancellation to get a representation theorem for preference relations on choices. However, the definition of the cancellation postulates for Savage acts and mixtures rely on (Savage) states. We now develop an analogue of this postulate for our syntactic notion of choice.

Definition 9. Given a set $T_{0}=\left\{t_{1}, \ldots, t_{n}\right\}$ of primitive tests, an atom over $T_{0}$ is a test of the form $t_{1}^{\prime} \wedge \ldots \wedge t_{n}^{\prime}$, where $t_{i}^{\prime}$ is either $t_{i}$ or $\neg t_{i}$.

An atom is a possible complete description of the truth value of tests according to the DM. If there are $n$ primitive tests in $T_{0}$, there are $2^{n}$ atoms. Let $\operatorname{At}\left(T_{0}\right)$ 
denote the set of atoms over $T_{0}$. It is easy to see that, for all tests $t \in T$ and atoms $\delta \in \operatorname{At}\left(T_{0}\right)$, and for all state spaces $S$ and standard test interpretations $\pi_{S}$, either $\pi_{S}(\delta) \subseteq \pi_{S}(t)$ or $\pi_{S}(\delta) \cap \pi_{S}(t)=\emptyset$. (The formal proof is by induction on the structure of $t$.) We write $\delta \Rightarrow t$ if the former is the case. We remark for future reference that a standard test interpretation is determined by its behavior on atoms. (It is, of course, also determined completely by its behavior on primitive tests).

Definition 10. An atom $\delta$ (resp., test $t$ ) is consistent with a theory $\mathcal{T}$ if there exists a state space $S$ and a test interpretation $\pi_{S} \in \Pi_{\mathcal{T}}$ such that $\pi_{S}(\delta) \neq \emptyset$ (resp., $\left.\pi_{S}(t) \neq \emptyset\right)$. Let $\operatorname{At}_{\mathcal{T}}\left(T_{0}\right)$ denote the set of atoms over $T_{0}$ consistent with $\mathcal{T}$.

Intuitively, an atom $\delta$ is consistent with $\mathcal{T}$ if there is some state in some state space where $\delta$ might hold, and similarly for a test $t$. The problem of checking whether an atom or a test is consistent with a theory $\mathcal{T}$ is NP-complete (it is basically the problem of testing whether a formula is satisfiable), that is, it is hard for the DM to do 3

A choice in $\mathcal{A}$ can be identified with a function from atoms to primitive choices in an obvious way. For example, if $a_{1}, a_{2}$, and $a_{3}$ are primitive choices and $T_{0}=$ $\left\{t_{1}, t_{2}\right\}$, then the choice $a=$ if $t_{1}$ then $a_{1}$ else (if $t_{2}$ then $a_{2}$ else $a_{3}$ ) can be identified with the function $f_{a}$ such that

- $f_{a}\left(t_{1} \wedge t_{2}\right)=f_{a}\left(t_{1} \wedge \neg t_{2}\right)=a_{1}$

- $f_{a}\left(\neg t_{1} \wedge t_{2}\right)=a_{2}$; and

- $f_{a}\left(\neg t_{1} \wedge \neg t_{2}\right)=a_{3}$.

Formally, we define $f_{a}$ by induction on the structure of choices. If $a \in \mathcal{A}_{0}$, then $f_{a}$ is the constant function $a$, and

$$
f_{\text {if } t \text { then } a \text { else } b}(\delta)= \begin{cases}f_{a}(\delta) & \text { if } \delta \Rightarrow t \\ f_{b}(\delta) & \text { otherwise }\end{cases}
$$

We consider a family of cancellation postulates, relativized to the axiom system $\mathcal{T}$. The cancellation postulate for $\mathcal{T}$ (given the language $\mathcal{A}_{0}$ ) is simply statewise cancellation for Savage acts, with atoms consistent with $\mathcal{T}$ playing the role of states.

\footnotetext{
${ }^{3}$ Satisfiability may or may not be relevant for the DM, depending on the task at hand.
} 
$\mathbf{A} 2^{\prime}$. If $\left\langle a_{1}, \ldots, a_{n}\right\rangle$ and $\left\langle b_{1}, \ldots, b_{n}\right\rangle$ are two sequences of choices in $\mathcal{A}_{\mathcal{A}_{0}, T_{0}}$ such that for each atom $\delta \in \operatorname{At}_{\mathcal{T}}\left(T_{0}\right),\left\{\left\{f_{a_{1}}(\delta), \ldots, f_{a_{n}}(\delta)\right\}\right\}=\left\{\left\{f_{b_{1}}(\delta), \ldots, f_{b_{n}}(\delta)\right\}\right\}$, and there exists some $k<n$ such that $a_{i} \succeq b_{i}$ for all $i \leq k, a_{k+1}=\cdots=a_{n}$, and $b_{k+1}=\cdots=b_{n}$, then $b_{n} \succeq a_{n}$.

We drop the prime and refer to A 2 when $k=n-1$.

Axiom A2 implies the simple cancellation of the last section, and so the conclusions of Proposition 1 hold: $\succeq$ on $\mathcal{A}$ will be transitive and reflexive. A2 has another consequence: a DM must be indifferent between $\mathcal{T}$-equivalent choices.

Proposition 5. Suppose that $\succeq$ satisfies A2. Then $a \equiv_{\mathcal{T}} b$ implies $a \sim b$.

Proposition [5] implies that the behavior of $a$ and $b$ on atoms not in $\operatorname{At}_{\mathcal{T}}\left(T_{0}\right)$ is irrelevant; that is, they are null in Savage's sense. We define this formally:

Definition 11. A test $t$ is null if, for all acts $a, b$ and $c$, if $t$ then $a$ else $c \sim$ if $t$ then $b$ else $c$.

An atom (or test) inconsistent with the theory $\mathcal{T}$ must be null (so we can test if the DM holds the theory $\mathcal{T}$ by checking whether, for all $\neg t \in \mathcal{T}$, $t$ is null), but consistent tests may be null as well. If we add as an axiom that no test consistent with the theory can be null, then (at least in principle) a DM's theory is testable.

The notion of a null test is suggestive of, more generally, test-contingent preferences.

Definition 12. If $t$ is a test in $T$, then for any acts $a$ and $b, a \succeq_{t} b$ iff for some $c$, if $t$ then $a$ else $c \succeq$ if $t$ then $b$ else $c$.

Proposition 3 shows that statewise cancellation implies that the choice of $c$ is irrelevant, and so test-contingent preferences are well-defined. Before giving our representation theorems, we compare our framework to that of Lipman (1999). Lipman starts with a collection of preference relations $\preceq_{I}$ indexed by what he calls information sets $I$. For Lipman, an information set is a possible piece of information that a DM might receive. An information set can be identified with a test in our framework (except that information sets do not have the syntactic structure of tests). Lipman takes 
a possible world to be a maximal piece of information; Lipman's possible worlds are essentially our atoms. Lipman takes the DM's subjective state space to be the set of possible worlds. As we show (Theorem 2), if we assume A1 (as Lipman does), then we can also take the DM's subjective state space in our representation to be the set of atoms consistent with the DM's theory $\mathcal{T}$. Otherwise, without $\mathrm{A} 1$, if we want a representation with a single utility function, we cannot in general take the state space to be just the set of atoms consistent with the DM's theory $\mathcal{T}$.

To get a representation theorem for $\mathcal{A}^{+}$, we use a mixture cancellation postulate, again replacing states by atoms. The idea now is that we can identify each choice $a$ with a function $f_{a}$ mapping atoms consistent with $\mathcal{T}$ into distributions over primitive choices. For example, if $t$ is the only test in $T_{0}$ and $\mathcal{T}=\emptyset$, then the choice $a=\frac{1}{2} a_{1}+\frac{1}{2}$ (if $t$ then $a_{2}$ else $a_{3}$ ) can be identified with the function $f_{a}$ such that

- $f_{a}(t)\left(a_{1}\right)=1 / 2 ; f_{a}(t)\left(a_{2}\right)=1 / 2$

- $f_{a}(\neg t)\left(a_{1}\right)=1 / 2 ; f_{a}(\neg t)\left(a_{3}\right)=1 / 2$.

Formally, we just extend the definition of $f_{a}$ given in the previous section by defining

$$
f_{r a_{1}+(1-r) a_{2}}(\delta)=r f_{a_{1}}(\delta)+(1-r) f_{a_{2}}(\delta) .
$$

Consider the following cancellation postulate:

$\mathbf{A} 2^{\dagger}$. If $\left\langle a_{1}, \ldots, a_{n}\right\rangle$ and $\left\langle b_{1}, \ldots, b_{n}\right\rangle$ are two sequences of acts in $\mathcal{A}_{\mathcal{A}_{0}, T_{0}}^{+}$such that

$$
f_{a_{1}}(\delta)+\cdots+f_{a_{n}}(\delta)=f_{b_{1}}(\delta)+\cdots+f_{b_{n}}(\delta)
$$

for all atoms $\delta$ consistent with $\mathcal{T}$, and there exists $k<n$ such that $a_{i} \succeq b_{i}$ for $i \leq k$, $a_{k+1}=\ldots=a_{n}$, and $b_{k+1}=\ldots=b_{n}$, then $b_{n} \succeq a_{n}$.

Again, $\mathrm{A} 2^{\dagger}$ can be viewed as a generalization of $\mathrm{A} 2^{\prime} 4$

\footnotetext{
${ }^{4} \mathbf{A 2}^{\dagger}$ is analogous to extended statewise mixture cancellation. It may seem strange that we need the cancellation to be statewise, since extended statewise mixture cancellation is equivalent to extended mixture cancellation. This suggests that we might be able to use a simpler non-statewise axiom. However, the obvious non-statewise version of the axiom and its statewise version are equivalent only if we assume that all that matters about a choice is how it acts as a function from atoms to primitive choices: If $f_{a}=f_{b}$, then $a \sim b$. Rather than add this axiom, we use $\mathbf{A 2}^{\dagger}$.
} 
We use $\mathrm{A}^{\dagger}$ or $\mathrm{A} 2^{\prime}$ in our representations theorems. Since we consider DMs who may have some obvious logical blindspots (and in Section 5 even consider DMs who may not satisfy the basic axioms of propositional logic), it may seem unreasonable to expect an agent to satisfy these postulates. We do not think it is always so unreasonable. For example, as we show in the proof of Theorem [5, if we assume completeness, in the Anscombe-Aumann setting, cancellation is equivalent to a number of other standard assumptions in the literature. Moreover, the preferences of a DM who is maximizing expected utility with respect to her subjective representation of the world will satisfy cancellation, even if the DM is not aware of the property and is not actively trying to ensure that her preferences are consistent with it. In any case, as we show, cancellation (or properties equivalent to it) is needed to get an SEU-like representation. So agents that do not satisfy it will simply not act as utility maximizers (even with respect to their subjective representation of the world).

\section{Representation Theorems}

Having discussed our framework, we now turn to the representation theorems. Our goal is to be as constructive as possible. In this spirit we want to require that preferences exist not for all possible acts that can be described in a given language, but only for those in a given subset, henceforth designated $C$. We are agnostic about the source of $C$. It could be the set of choices in one particular decision problem, or it could be the set of choices that form a universe for a collection of decision problems. One cost of our finite framework is that we will have no uniqueness results. In our framework the preference representation can fail to be unique because of our freedom to choose different state and outcome spaces, but even given these choices, the lack of richness of $C$ may allow multiple representations of the same (partial) order.

\subsection{A Representation Theorem for $\mathcal{A}$}

By a representation for a preference relation on $\mathcal{A}$ we mean the following:

Definition 13. A preference relation on a set $C \subseteq \mathcal{A}_{\mathcal{A}_{0}, T_{0}}$ has a constructive $\mathcal{T}$ consistent SEU representation iff there is a finite set of states $S$, a finite set $O$ of outcomes, a set $\mathcal{U}$ of utility functions $u: O \rightarrow \mathbf{R}$, a set $\mathcal{P}$ of probability distributions 
on $S$, a subset $\mathcal{V} \subseteq \mathcal{U} \times \mathcal{P}$, a test interpretation $\pi_{S}$ consistent with $\mathcal{T}$, and a choice interpretation $\rho_{S O}$ such that $a \succeq b$ iff

$$
\sum_{s \in S} u\left(\rho_{S O}(a)(s)\right) p(s) \geq \sum_{s \in S} u\left(\rho_{S O}(b)(s)\right) p(s) \text { for all }(u, p) \in \mathcal{V}
$$

We are about to claim that $\succeq$ satisfies $A 2^{\prime}$ if and only if it has a constructive $\mathcal{T}$-consistent representation. In the representation, we have a great deal of flexibility as to the choice of the state space $S$ and the outcome space $O$. One might have thought that the space of atoms, $\operatorname{At}_{\mathcal{T}}\left(T_{0}\right)$, would be a rich enough state space on which to build representations. This is not true when preferences are incomplete and we ask for a representation with a single utility function. A rich enough state space is needed to account for the incompleteness.

Definition 14. Given a partial order $\succeq$ on a set $C$ of choices, let $E X_{\mathrm{AX}}(\succeq)$ denote all the extensions of $\succeq$ to a total order on $C$ satisfying A2.

Our proof shows that we can take $S$ to be $\operatorname{At}_{\mathcal{T}}\left(T_{0}\right) \times E X_{\mathrm{AX}}(\succeq)$. Thus, in particular, if $\succeq$ is complete, then we can take the state space to be $\operatorname{At}_{\mathcal{T}}\left(T_{0}\right)$. We later give examples that show that if $\succeq$ is not complete then, in general, if we ask for a representation with a single utility function, then the state space must have cardinality larger than that of $\operatorname{At}_{\mathcal{T}}\left(T_{0}\right)$. While for some applications there may be a more natural state space, our choice of state space shows that we can always view the DM's uncertainty as stemming from two sources: the truth values of various tests (which are determined by the atoms) and the relative order of two choices not determined by $\succeq$ (which is given by the extension $\succeq^{\prime} \in E X_{\mathrm{AX}}(\succeq)$ of $\succeq$ ). The idea of a DM being uncertain about her preferences is prevalent elsewhere in decision theory; for instance, in the menu choice literature (Kreps, 1979). This uncertainty can be motivated in any number of ways, including both incomplete information and resource-bounded reasoning.

Theorem 2. A preference relation $\succeq$ on a set $C \subseteq \mathcal{A}_{\mathcal{A}_{0}, T_{0}}$ has a constructive $\mathcal{T}$ consistent SEU representation iff $\succeq$ satisfies A2'. Moreover, in the representation, either $\mathcal{P}$ or $\mathcal{U}$ can be taken to be a singleton and, if $\mathcal{U}$ is a singleton $\{u\}$, the state space can be taken to be $\operatorname{At}_{\mathcal{T}}\left(T_{0}\right) \times E X_{\mathrm{AX}}(\succeq)$. If, in addition, $\succeq$ satisfies $\boldsymbol{A} 1$, then $\mathcal{V}$ can be taken to be a singleton (i.e., both $\mathcal{P}$ and $\mathcal{U}$ can be taken to be singletons).

Theorem 2 is proved in the appendix. The proof proceeds by first establishing a statedependent representation using $\mathcal{A}_{0}$ as the outcome space, and then, by changing 
the outcome space, 'bootstrapping' the representation to an EU representation. This technique shows that, when the state and outcome spaces are part of the representation, there is no difference between the formal requirement for a state-dependent representation and that for a SEU representation. This does not mean that expected utility comes 'for free'; rather, we interpret it to mean that the beliefs/desires formalism that motivates expected utility theory is sensible for the decision problems discussed in this subsection only if the particular outcome space chosen for the representation has some justification external to our theory. We note that if preferences satisfy A1, the theorem requires only the cancellation axiom A2 rather than the stronger $\mathrm{A} 2{ }^{\prime}$.

There are no uniqueness requirements on $\mathcal{P}$ or $\mathcal{U}$ in Theorem 2. In part, this is because the state space and outcome space are not uniquely determined. But even if $\mathbf{A} \mathbf{1}$ holds, so that the state space can be taken to be the set of atoms, the probability and the utility are far from unique, as the following example shows.

Example 6. Take $\mathcal{A}_{0}=\{a, b\}, T_{0}=\{t\}$, and $\mathcal{T}=\emptyset$. Suppose that $\succeq$ is the reflexive transitive closure of the following string of preferences:

$$
a \succ \text { if } t \text { then } a \text { else } b \succ \text { if } t \text { then } b \text { else } a \succ b \text {. }
$$

Every choice in $\mathcal{A}$ is equivalent to one of these four, so $\mathbf{A} 1$ holds, and we can take the state space to be $S^{*}=\{t, \neg t\}$. Let $O^{*}=\left\{o_{1}, o_{2}\right\}$, and define $\rho_{S^{*} O^{*}}^{0}$ so that $\rho_{S^{*} O^{*}}^{0}(a)$ is the constant function $o_{1}$ and $\rho_{S^{*} O^{*}}^{0}(b)$ is the constant function $o_{2}$. Now define $\pi_{S^{*}}$ in the obvious way, so that $\pi_{S^{*}}(t)=\{t\}$ and $\pi_{S^{*}}(\neg t)=\{\neg t\}$. We can represent the preference relation by using any probability measure $p^{*}$ such that $p^{*}(t)>1 / 2$ and any utility function $u^{*}$ such that $u^{*}\left(o_{1}\right)>u^{*}\left(o_{2}\right)$.

As Example 6 shows, the problem really is that the set of actions is not rich enough to determine the probability and utility. By way of contrast, Savage's postulates ensure that the state space is infinite and that there are at least two outcomes. Since the acts are all functions from states to outcomes, there must be uncountably many acts in Savage's framework.

The next example shows that without the completeness axiom A1, there may be no representation in which there is only one utility function and the state space is $\operatorname{At}_{\mathcal{T}}\left(T_{0}\right)$.

Example 7. Suppose that $T_{0}=\emptyset, \mathcal{T}=\emptyset$, and $\mathcal{A}_{0}$ (and hence $\mathcal{A}$ ) consists of the two primitive choices $a$ and $b$, which are incomparable. In this case, the smallest state 
space that we can use has cardinality at least 2 . For if $|S|=1$, then there is only one possible probability measure on $S$, so $a$ and $b$ cannot be incomparable. Since there is only one atom when there are no primitive propositions, we cannot take the state space to be the set of atoms. There is nothing special about taking $T_{0}=\emptyset$ here; similar examples can be constructed showing that we cannot take the state space to be $\operatorname{At}_{\mathcal{T}}\left(T_{0}\right)$ for arbitrary choices of $T_{0}$ if the preference relation is partial. An easy argument also shows that there is no representation where $|O|=1$.

This preference relation can be represented with two outcomes and two states. Let $S=\left\{s_{1}, s_{2}\right\}$ and $O=\left\{o_{1}, o_{2}\right\}$. Define $\rho_{S O}^{0}(a)\left(s_{i}\right)=o_{i}$, and $\rho_{S O}^{0}(b)\left(s_{i}\right)=o_{2-i}$ for $i=1,2$. Let $\mathcal{U}$ contain a single function such that $u\left(o_{1}\right) \neq u\left(o_{2}\right)$. Let $\mathcal{P}$ be any set of probability measures including the measures $p_{1}$ and $p_{2}$ such that $p_{1}\left(s_{1}\right)=1$ and $p_{2}\left(s_{1}\right)=0$. Then the expected utility ranking of randomized acts under each $p_{i}$ contains no nontrivial indifference, and the ranking under $p_{2}$ is the reverse of that under $p_{1}$. Thus, these choices represent the preference relation.

The assumption that there is only one utility function is critical here. For example, we could also represent this preference relation using a single state and two utility functions, $u_{1}$ and $u_{2}$, where $u_{1}(a)>u_{1}(b)$ and $u_{2}(b)>u_{2}(a)$. We conjecture that if we allow multiple utility functions, then there is always a representation where the state space is $\operatorname{At}_{\mathcal{T}}\left(T_{0}\right)$.

\subsection{A Representation Theorem for $\mathcal{A}^{+}$}

The purpose of this subsection is to show that for the language $\mathcal{A}^{+}$, we can get something much in the spirit of the standard representation theorem for AA acts. The standard representation theorem has a mixture independence axiom and an Archimedean axiom. As we have seen, $\mathrm{A}^{\dagger}$ gives us rational mixture independence; it does not suffice for full mixture independence. To understand what we need, recall that the standard Archimedean axiom for AA acts has the following form:

Arch. If $a \succ b \succ c$ then there exist $r, r^{\prime} \in(0,1)$ such that $a \succ r a+(1-r) c \succ b \succ$ $r^{\prime} a+\left(1-r^{\prime}\right) c \succ c$.

While this axiom is both necessary for and implied by the existence of an SEU representation when $\succeq$ is complete, the following example describes an incomplete 
preference relation $\succeq$ with a multi-probability SEU representation that fails to satisfy Arch.

Example 8. Suppose that $S=\left\{s_{1}, s_{2}, s_{3}\right\}$. Let $a_{1}, a_{2}$, and $a_{3}$ be acts such that $a_{i}\left(s_{j}\right)$ gives an outcome of 1 if $i=j$ and 0 otherwise. Let $\mathcal{P}$ consist of all probability distributions $p$ on $S$ such that $p\left(s_{1}\right) \geq p\left(s_{2}\right) \geq p\left(s_{3}\right)$. Define $\succeq$ by taking $a \succeq b$ iff the expected outcome of $a$ is at least as large as that of $b$ with respect to all the probability distributions in $\mathcal{P}$. It is easy to see that $a_{1} \succ a_{2} \succ a_{3}$, but for no $r \in(0,1)$ is it the case that $r a_{1}+\left(1-r_{1}\right) a_{3} \succ a_{2}$ (consider the probability distribution $p$ such that $\left.p\left(s_{1}\right)=p\left(s_{2}\right)=1 / 2\right)$.

We can think of the Archimedean axiom as trying to capture some continuity properties of $\succeq$. We use instead the following axiom, which was also used by Aumann (1962, 1964). If the set of tests has cardinality $n$ and the set of primitive choices has cardinality $m$, we can identify an act $\mathcal{A}^{+}$with an element of $\mathbf{R}^{\mathbf{2}^{\mathbf{n}} \mathbf{m}}$, so the graph of $\succeq$ (i.e., the set of pairs $(x, y)$ such that $x \succeq y$ ) can be viewed as a subset of $\mathbf{R}^{2^{\mathbf{n}+1}} \mathbf{m}$.

A3. The graph of the preference relation $\succeq$ is closed.

As the following result shows, in the presence of $\mathrm{A}^{\dagger}$ (extended statewise mixture cancellation), A3 implies full mixture independence. Moreover, if we also assume A1, then A3 implies Arch. Indeed, it will follow from Theorem 3 that in the presence of $\mathbf{A} 1$ and $\mathbf{A} 2^{\dagger}, \mathbf{A} 3$ and Arch are equivalent. On the other hand, it seems that Arch does not suffice to capture independence if $\succeq$ is a partial order. Summarizing, A3 captures the essential features of the Archimedean property, while being more appropriate if $\succeq$ is only a partial order.

Proposition 6. (a) $\mathrm{A} 2^{\dagger}$ and A3 imply full mixture independence.

(b) A1, A3, and extended mixture cancellation together imply Arch.

Definition 15. A preference relation $\succeq$ on a set $C \subseteq \mathcal{A}_{\mathcal{A}_{0}, T_{0}}^{+}$has a constructive $\mathcal{T}$ consistent SEU representation iff there is a finite set of states $S$, a finite set $O$ of outcomes, a set $\mathcal{U}$ of utility functions $u: O \rightarrow \mathbf{R}$, a closed set $\mathcal{P}$ of probability distributions on $S$, a closed set $\mathcal{V} \subseteq \mathcal{U} \times \mathcal{P}$, a test interpretation $\pi_{S}$ consistent with $\mathcal{T}$, and a choice interpretation $\rho_{S O}$ such that $a \succeq b$ iff

$$
\sum_{s, o} u(o) \rho_{S O}(a)(s)(o) p(s) \geq \sum_{s, o} u(o) \rho_{S O}(b)(s)(o) p(s) \text { for all }(u, p) \in \mathcal{V}
$$


In the statement of the theorem, if $\succeq$ is a preference relation on a mixtureclosed subset of $\mathcal{A}_{\mathcal{A}_{0}, T_{0}}^{+}$, we use $\succeq \otimes(a, b)$ to denote the smallest preference relation including $\succeq$ and $(a, b)$ satisfying $\mathbf{A} 2^{\dagger}$ and $\mathbf{A} 3$, and take $E X_{\mathrm{AX}}^{+}(\succeq)$ to consist of all complete preference relations extending $\succeq$ and satisfying $\mathrm{A} 2^{\dagger}$ and A3.

Theorem 3. A preference relation $\succeq$ on a closed and mixture-closed set $C \subseteq \mathcal{A}_{\mathcal{A}_{0}, T_{0}}^{+}$ has a constructive $\mathcal{T}$-consistent SEU representation iff $\succeq$ satisfies $\mathrm{A} 2^{\dagger}$ and $\mathrm{A} 3$. Moreover, in the representation, either $\mathcal{P}$ or $\mathcal{U}$ can be taken to be a singleton and, if $\mathcal{U}$ is a singleton $\{u\}$, the state space can be taken to be $\operatorname{At}_{\mathcal{T}}\left(T_{0}\right) \times E X_{\mathrm{AX}}^{+}(\succeq)$. If, in addition, $\succeq$ satisfies $\boldsymbol{A 1}$, then $\mathcal{V}$ can be taken to be a singleton.

As in the case of the language $\mathcal{A}$, we cannot in general take the state space to be the set of atoms. Specifically, if $\mathcal{A}_{0}$ consists of two primitive choices and we take all choices in $\mathcal{A}_{0}^{+}$to be incomparable, then the same argument as in Example 7 shows that we cannot take $S$ to be $\operatorname{At}\left(T_{0}\right)$, and there are no interesting uniqueness requirements that we can place on the set of probability measures or the utility function. On the other hand, if $\mathbf{A} 1$ holds, the proof of Theorem 3 shows that, in the representation, the expected utility is unique up to affine transformations. That is, if $\left(S, O, p, \pi_{S}, \rho_{S O}^{0}, u\right)$ and $\left(S^{\prime}, O^{\prime}, p^{\prime}, \pi_{S^{\prime}}, \rho_{S^{\prime} O^{\prime}}^{0}, u^{\prime}\right)$ are both representations of $\succeq$, then there exist constants $\alpha$ and $\beta$ such that for all acts $a \in \mathcal{A}_{\mathcal{A}_{0}, T_{0}}^{+}$, $\mathrm{E}_{p}\left(u\left(\rho_{S O}(a)\right)\right)=\alpha \mathrm{E}_{p^{\prime}}\left(u^{\prime}\left(\rho_{S^{\prime} O^{\prime}}(a)\right)\right)+\beta$.

\subsection{Objective Outcomes}

In choosing, for instance, certain kinds of insurance or financial assets, there is a natural, or objective, outcome space-in these cases, monetary payouts. To model this, we take the set $O$ of objective outcomes as given, and identify it with a subset of the primitive acts $A_{0}$. Call the languages with this distinguished set of outcomes $\mathcal{A}_{\mathcal{A}_{0}, T_{0}, O}$ and $\mathcal{A}_{\mathcal{A}_{0}, T_{0}, O}^{+}$, depending on whether we allow randomization.

To get a representation theorem in this setting, we need to make some standard assumptions. The first is that there is a best and worst outcome; the second is a state-independence assumption. However, this state-independence assumption only applies to acts in $O$, but not to all acts.

A4. There are outcomes $o_{1}$ and $o_{0}$ such that for all non-null tests $t, o_{1} \succeq_{t} a \succeq_{t} o_{0}$ for all $a \in \mathcal{A}_{0}$. 
A5. If $t$ is not null and $o, o^{\prime} \in O$, then $o \succeq o^{\prime}$ iff $o \succeq_{t} o^{\prime}$.

In all our earlier representation theorems, it was possible to use a single utility function. A4 and A5 do not suffice to get such a representation theorem. A necessary condition to have a single utility function, if we also want utility to be state independent, is that $\succeq$ restricted to $O$ be complete.

A6. $\succeq$ restricted to $O$ is complete.

While A5 and A6 are necessary to get a representation with a single utility function, they are not sufficient, as the following example shows.

Example 9. Suppose that we have a language with one primitive test $t$, and three outcomes, $o_{0}, o_{1}$, and $o$. Let $a_{1}$ be if $t$ then $o_{0}$ else $o_{1}$ and let $a_{2}$ be if $t$ then $o_{1}$ else $o_{0}$. Let $\succeq$ be the smallest preference relation satisfying $\mathrm{A} 2^{\prime}, \mathrm{A} 4, \mathrm{~A} 5$, and $\mathrm{A} 6$ (or $\mathrm{A} 2^{\dagger}$, A3, A4, A5, and A6, if we are considering the language $\mathcal{A}^{+}$) such that $o \sim a_{1}$ and $o_{1} \succ o_{0}$. Note that $a_{1}$ and $a_{2}$ are incomparable according to $\succeq$. Suppose that there were a representation of $\succeq$ involving a set $\mathcal{P}$ of probability measures and a single utility function $u$. Thus, there would have to be probability measures $p_{1}$ and $p_{2}$ in $\mathcal{P}$ such that $a_{1} \succ a_{2}$ according to $\left(p_{1}, u\right)$ and $a_{2} \succ a_{1}$ according to $\left(p_{2}, u\right)$. It easily follows that $p_{1}\left(\pi_{S}(t)\right)<1 / 2$ and $p_{2}\left(\pi_{S}(t)\right)>1 / 2$. We can assume without loss of generality (by using an appropriate affine transformation) that $u\left(o_{0}\right)=0$ and $u\left(o_{1}\right)=1$. Since $o \sim a_{1}, u(o)$ must be the same as the expected utility of $a_{1}$. But this expected utility is less than $1 / 2$ with $p_{1}$ and more than $1 / 2$ with $p_{2}$. This gives the desired contradiction.

Part of the problem is that it is not just the acts in $O$ that must be state independent. Let $O^{+}$be the smallest set of acts containing $O$ that is closed under convex combinations, so that if $o$ and $o^{\prime}$ are in $O^{+}$, then so is $r o+(1-r) o^{\prime}$. Let $\mathbf{A} 5^{+}$and $\mathbf{A 6}^{+}$be the axioms that result by replacing $O$ by $O^{+}$in $\mathbf{A} 5$ and $\mathbf{A 6}$, respectively. Example 9 actually shows that $\mathrm{A}^{+}$and $\mathrm{A} 6$ do not suffice to get a single utility function; Theorem 4 shows that $\mathrm{A}^{+}$and $\mathrm{A6}^{+}$do, at least for $\mathcal{A}^{+}$. We do not have a representation theorem for $\mathcal{A}$, and believe it will be hard to obtain such a theorem (for much the same reasons that it is hard to get a representation theorem in the Savage setting if we restrict to a finite set of acts).

Theorem 4. A preference relation $\succeq$ on a set $C \subseteq \mathcal{A}_{\mathcal{A}_{0}, T_{0}, O}^{+}$has a constructive SEU representation with outcome space $O$ iff $\succeq$ satisfies $\mathbf{A} 2^{\dagger}, \mathbf{A} 3, \mathbf{A} 4, \mathbf{A} 5^{+}$, and $\mathbf{A} 6^{+}$. 
Moreover, in the representation, $\mathcal{U}$ can be taken to be a singleton $\{u\}$ and the state space can be taken to have the form $\operatorname{At}_{\mathcal{T}}\left(T_{0}\right) \times \mathcal{A}_{0} \times E X_{\mathrm{AX}}^{+}(\succeq)$. If in addition $\succeq$ satisfies $\boldsymbol{A 1}$, then we can take $\mathcal{V}$ to be a singleton too.

Note that, even if $\succeq$ satisfies $\mathbf{A} 1$, the state space has the form $\operatorname{At}_{\mathcal{T}}\left(T_{0}\right) \times \mathcal{A}_{0}$. The fact that we cannot take the state space to be $\operatorname{At}_{\mathcal{T}}\left(T_{0}\right)$ is a consequence of our assumption that primitive acts are deterministic. Roughly speaking, we need the extra information in states to describe our uncertainty regarding how the primitive acts not in $O$ can be viewed as functions from states to outcomes in the pre-specified set $O$. The following example shows that we need the state space to be larger than $\operatorname{At}_{\mathcal{T}}\left(T_{0}\right)$ in general.

Example 10. Suppose that there are no primitive propositions, so $\operatorname{At}_{\mathcal{T}}\left(T_{0}\right)$ is a singleton. There are three primitive acts in $\mathcal{A}_{0}: o_{1}=\$ 50000, o_{0}=\$ 0$, and $a$, which is interpreted as buying 100 shares of Alphabet. Suppose that $o_{1} \succ a \succ o_{0}$. If there were a representation with only one state, then $\rho_{S O}(a)$ would have to be either $o_{1}$ or $o_{0}$, which would imply that $a \sim o_{1}$ or $a \sim o_{0}$, contradicting our description of $\succeq$. The issue here is our requirement that a primitive choice be represented as a function from states to outcomes. If we could represent $a$ as a lottery, there would be no problem representing $\succeq$ with one state. We could simply take $u\left(o_{1}\right)=1, u\left(o_{0}\right)=0$, and take $a$ to be a lottery that gives each of $o_{0}$ and $o_{1}$ with probability $1 / 2$.

We prefer not to allow the DM to consider such 'subjective' lotteries. Rather, we have restricted the randomization to acts. The representation theorem would not change if we allowed primitive choice to map a state to a distribution over outcomes, rather than requiring them to be mappings from states to single outcomes (except that we could take the state space to be $\operatorname{At}_{\mathcal{T}}\left(T_{0}\right)$ ).

We can easily represent $\succeq$ using two states, $s_{0}$ and $s_{1}$, by taking $a$ to be the act with outcome $o_{i}$ in state $s_{i}$, for $i=0,1$. Taking each of $s_{0}$ and $s_{1}$ to hold with probability $1 / 2$ then gives a representation of $\succeq$. In this representation, we can view $s_{0}$ as the state where buying Alphabet is a good investment, and $s_{1}$ as the state where buying Alphabet is a bad investment. However, the DM cannot talk about Alphabet being a good investment; this is not part of his language. Another DM might explicitly consider the test that Alphabet is a good investment. Suppose this DM has the same preference relation over primitive acts as in the example, is indifferent between 100 shares of Alphabet and $o_{1}$ if the test is true, and is indifferent between 100 shares of Alphabet and $o_{0}$ if the test is false. This DM's preference relation has exactly the 
same representation as the first DM's preference relation, but now $s_{0}$ can be viewed as the atom where the test is false, and $s_{1}$ can be viewed as the atom where the test is true. The second DM can reason about Alphabet being a good investment explicitly, and can talk to others about it.

\section{Nonstandard Interpretations}

Failures of extensionality are concerned with a semantic issue: Identifying when two descriptions of an event are equivalent. However, the same issue arises with respect to logical equivalence. For instance, suppose act $a$ gives a DM $x$ if $\neg\left(\left(\neg t_{1} \wedge \neg t_{3}\right) \vee\right.$ $\left.\left(\neg t_{2} \wedge \neg t_{3}\right)\right)$ is true, and $y$ otherwise, and act $b$ gives the DM $x$ if $\left(t_{1} \wedge t_{2}\right) \vee t_{3}$ is true, and $y$ otherwise. Only someone adept at formula manipulation (or with a good validity-checking program) will recognize that acts $a$ and $b$ are equivalent as a matter of logic because the equivalence of the two compound propositions is a tautology. So far we have required that DMs be logically omniscient, and recognize all such tautologies, because we have considered only standard interpretations.

An interpretation $\pi_{S}$ on a state space $S$ does not have to be standard; all that is required is that it associate with each test a subset of $S$. By allowing nonstandard test interpretations, we can back off from our requirement that DM's know all tautologies. This gives us a way of modeling failures of logical omniscience and, in particular, resource-bounded reasoning by DMs. We remark that such nonstandard interpretations are essentially what philosophers call 'impossible possible worlds' (Rantala, 1982); they have also been used in game theory for modeling resourcebounded reasoning (Lipman, 1999).

A standard test interpretation is completely determined by its behavior on the primitive tests. However, in general, there is no similar finite characterization of a nonstandard test interpretation. To keep things finite, when dealing with nonstandard interpretations, we assume that there is a finite subset $T^{*}$ of the set $T$ of all tests such that the only tests that appear in choices are those in $T^{*}$. (This is one way to model resource-bounded reasoning.) With this constraint, it suffices to consider the behavior of a nonstandard interpretations only on the tests in $T^{*}$. Let $\mathcal{A}_{\mathcal{A}_{0}, T^{*}}$ consist of all choices whose primitive choices are in $\mathcal{A}_{0}$ and whose tests are all in $T^{*}$.

The restriction to choices in $\mathcal{A}_{\mathcal{A}_{0}, T^{*}}$ allows us to define the cancellation pos- 
tulate in a straightforward way even in the presence of nonstandard interpretations. A truth assignment to $T^{*}$ is just a function $v: T^{*} \rightarrow\{$ true, false $\}$. We can identify an interpretation on $S$ with a function that associates with every state $s \in S$ the truth assignment $v_{s}$ such that $v_{s}(t)=$ true iff $s \in \pi(t)$. For a standard interpretation, we can use an atom instead of a truth assignment, since for a standard interpretation, the behavior of each truth assignment is determined by its behavior on primitive propositions, and we can associate with the truth assignment $v_{s}$ that atom $\delta_{s}$ such that $t$ is a conjunct in $\delta_{s}$ iff $v_{s}(t)=$ true. These observations suggest that we can consider truth assignments to be the generalization of atoms once we move to nonstandard interpretations. Indeed, if we do this, we can easily generalize all our earlier theorems.

In more detail, we now view a choice as a function, not from atoms to primitive choices, but, more generally, as a function from truth assignments to primitive choices. As before, we take primitive choices to be constant functions. The choice $a=$ if $t_{1}$ then $a_{1}$ else (if $t_{2}$ then $a_{2}$ else $a_{3}$ ) can be identified with the function $f_{a}$ such that

and

$$
f_{a}(v)= \begin{cases}a_{1} & \text { if } v\left(t_{1}\right)=\text { true } \\ f_{\text {if } t_{2} \text { then } a_{2} \text { else } a_{3}} & \text { if } v\left(t_{1}\right)=\text { false }\end{cases}
$$

$$
f_{\text {if } t_{2}} \text { then } a_{2} \text { else } a_{3}= \begin{cases}a_{2} & \text { if } v\left(t_{2}\right)=\text { true } \\ a_{3} & \text { if } v\left(t_{2}\right)=\text { false. }\end{cases}
$$

A truth assignment $v$ is consistent with $\mathcal{T}$ if $v(t)=$ true for all tests $t \in \mathcal{T}$.

With these definitions in hand, all our earlier results hold, with the following changes:

- we replace $\mathcal{A}_{\mathcal{A}_{0}, T_{0}}$ by $\mathcal{A}_{\mathcal{A}_{0}, T^{*}}$;

- we replace 'atoms $\delta$ over $T_{0}$ ' by 'truth assignment to $T^{*}$.

The cancellation axioms are all now well defined. With these changes, Proposition [5 and Theorems 2, 3, and 4 hold with essentially no changes in the proof. Thus, we have representation theorems that apply even to resource-bounded reasoners.

There is one further subtlety, however. A theory puts constraints on the set of test interpretations we consider. Up to now, we have taken a theory $\mathcal{T}$ to be a 
collection of tests. If we restrict to standard interpretations, this suffices, in the sense that, given a state space $S$, for all sets $\mathcal{I}$ of standard interpretations for the state space $S$ of a set $T$ of tests, there is a theory $\mathcal{T}_{\mathcal{I}}$ such that a test interpretation $\pi_{S}$ for $S$ respects $\mathcal{T}_{\mathcal{I}}$ iff $\pi_{S} \in \mathcal{I}$. That is, a theory can specify a set of interpretations. This is no longer the case once we move to nonstandard interpretations. For example, the nonstandard interpretation $\pi_{S}^{*}$ for $S$ that makes every test true at every state (i.e., $\pi_{S}^{*}(t)=S$ for all tests $t$ ) respects every theory. There is no way that a theory can disallow $\pi_{S}^{*}$.

To understand why this is an issue, note that with standard interpretations, we get many properties for free, so to speak. For example, for all tests $t, t$ and $\neg t$ cannot be simultaneously true. More precisely, for all standard interpretations $\pi_{S}$ for $S$, we have $\pi_{S}(t) \cap \pi_{S}(\neg t)=\emptyset$. We might want to restrict to nonstandard interpretations that have this property. Unfortunately, there is no way to do this if a theory is just a set of tests; we cannot exclude the interpretation $\pi_{S}^{*}$, and it does not have this property. Similarly, we may want to restrict to interpretations where conjunction is commutative, so $\pi_{S}\left(t_{1} \wedge t_{2}\right)=\pi_{S}\left(t_{2} \wedge t_{1}\right)$. Note that just adding the test $\left(t_{1} \wedge t_{2}\right) \Leftrightarrow\left(t_{2} \wedge t_{1}\right)$ does not have this effect; it just ensures that $\pi_{S}\left(\left(t_{1} \wedge t_{2}\right) \Leftrightarrow\left(t_{2} \wedge t_{1}\right)\right)=S$.

We thus consider generalized theories, which consist of generalized axioms of the form $T_{1}=T_{2}$, where $T_{1}$ and $T_{2}$ are sets of tests. We also assume that there are special primitive tests true and false. A test interpretation $\pi_{S}$ for a state space $S$ respects a generalized theory $\mathcal{T}$ if, for every generalized axiom $T_{1}=T_{2}$ in $\mathcal{T}$, $\left\{s \in S: s \in \pi_{S}(t)\right.$ for all $\left.t \in T_{1}\right\}=\left\{s \in S: s \in \pi_{S}(t)\right.$ for all $\left.t \in T_{2}\right\}$; that is, we want all the tests in $T_{1}$ to be true at a state $s$ iff all the tests in $T_{2}$ are true. Moreover, we require that $\pi_{S}($ true $)=S$ and $\pi_{S}($ false $)=\emptyset$.

Working with generalized theories gives us a great deal more power to put constraints on nonstandard interpretations. For example:

- If $\mathcal{T}$ contains the generalized axiom $T=\{$ true $\}$, then all the tests in $T$ are true at every state of a nonstandard interpretation that respects $\mathcal{T}$. (Thus, this particular generalized axiom has the same effect as the theories we considered earlier.)

- If $\mathcal{T}$ contains $\left\{t_{1} \wedge t_{2}\right\}=\left\{t_{2} \wedge t_{1}\right\}$ for all tests $t_{1}$ and $t_{2}$, then conjunction is commutative for all nonstandard interpretations that respect $\mathcal{T}$. 
- If $\mathcal{T}$ contains $\{t, \neg t\}=\{F\}$, then at most one of $t$ and $\neg t$ will be true according to $\pi_{S}$.

Despite their added expressive power (once we allow nonstandard interpretations), Proposition [5 and Theorems 2,3 , and 4 continue to hold with essentially no changes in the proof if we allow generalized theories.

\section{Updating}

There is nothing unique about the state space chosen for an SEU representation of a given choice problem. Our representation theorems state that if an SEU representation exists on any given state space and outcome space with test and choice interpretation functions, then preferences satisfy the appropriate cancellation and other appropriate axioms. Our proofs, however, show that (for standard interpretations) we can always represent a choice situation on the state space $\operatorname{At}_{\mathcal{T}}\left(T_{0}\right) \times E X_{\mathrm{AX}}(\succeq)$, or $\operatorname{At}_{\mathcal{T}}\left(T_{0}\right) \times \mathcal{A}_{0} \times E X_{\mathrm{AX}}(\succeq)$ for the objective-outcomes case, so this construction is in some sense canonical. Just as importantly, this state space respects the DM's choice of language and her preferences, and seems like a natural state space for the $\mathrm{DM}$ to use when reasoning about the decision problem.

In our models, there are two kinds of information. A DM can learn more about the external world, that is, learn the results of some tests. A DM can also learn more about her internal world, that is, she can learn more about her preferences. This learning takes the form of adding more comparisons to her (incomplete) preference relation. To make this precise, given a preference relation $\succeq$ on a set $C \subseteq \mathcal{A}_{\mathcal{A}_{0}, T_{0}}$ satisfying $\mathbf{A} 2^{\prime}$, let $\succeq \oplus(a, b)$ be the smallest preference relation including $\succeq$ and $(a, b)$ satisfying $\mathbf{A} 2^{\prime}$. (There is such a smallest preference relation, since it is easy to see that if $\succeq^{\prime}$ and $\succeq^{\prime \prime}$ both extend $\succeq$, include $(a, b)$, and satisfy $\mathbf{A} 2^{\prime}$, then so does $\succeq^{\prime} \cap \succeq^{\prime \prime}$.)

If we take the state space to be $\operatorname{At}_{\mathcal{T}}\left(T_{0}\right) \times E X_{\mathrm{AX}}(\succeq)$, then a DM's preference relation after obtaining either new test information or new comparison information can be represented by conditioning the original probability measures. If $\mathcal{P}$ is a set of probability distributions on some set $S$ and $E$ is a measurable subset of $S$, let $\mathcal{P} \mid E=\{q: q=p(\cdot \mid E)$ for some $p \in \mathcal{P}$ with $p(E)>0\}$. That is, in computing 
$\mathcal{P} \mid E$, we throw out all distributions $p$ such that $p(E)=0$, and then apply standard conditioning to the rest. Let $\mathcal{P}|t=\mathcal{P}| \pi_{S}(t)$. In the theorems below, we condition on a test $t$ and on a partial order $\succeq^{\prime}$ extending $\succeq$. We are implicitly identifying $t$ with the event $\left\{\delta \in \operatorname{At}_{\mathcal{T}}\left(T_{0}\right): \delta \Rightarrow t\right\}$, and $\succeq^{\prime}$ with the set of total orders in $E X_{\mathrm{AX}}(\succeq)$ extending $\succeq$.

Theorem 2 $\mathbf{c}$. Under the assumptions of Theorem 2, and with a representation of $\succeq$ in which $S=\operatorname{At}_{\mathcal{T}}\left(T_{0}\right) \times E X_{\mathrm{AX}}(\succeq)$ and $\mathcal{U}$ is a singleton $\{u\}$, $\succeq_{t}$ is represented by $\mathcal{P} \mid t$ and $u$, and $\succeq \oplus(a, b)$ is represented by $\mathcal{P} \mid(\succeq \oplus(a, b))$ and $u$.

Theorem $3 \mathbf{c}$. Under the assumptions of Theorem 3 , and with a representation of $\succeq$ in which $S=\operatorname{At}_{\mathcal{T}}\left(T_{0}\right) \times E X_{\mathrm{AX}}^{+}(\succeq)$ and $\mathcal{U}$ is a singleton $\{u\}, \succeq_{t}$ is represented by $\mathcal{P} \mid t$ and $\{u\}$, and $\succeq \otimes(a, b)$ is represented by $\mathcal{P} \mid(\succeq \otimes(a, b))$ and $u$.

Theorem 4 $\mathbf{c}$. Under the assumptions of Theorem 4, and with a representation of $\succeq$ in which $S=\operatorname{At}_{\mathcal{T}}\left(T_{0}\right) \times \mathcal{A}_{0} \times E X_{\mathrm{AX}}^{+}(\succeq)$ and $\mathcal{U}$ is a singleton $\{u\}$, $\succeq_{t}$ is represented by $\mathcal{P} \mid t$ and $u$, and $\succeq \otimes(a, b)$ is represented by $\mathcal{P} \mid(\succeq \otimes(a, b))$ and $u$.

Information in the external world is modeled as a restriction on the set of feasible acts; information in the internal world is adding comparisons to a the preference relation. These theorems show that both kinds of information can be modeled within a Bayesian paradigm.

\section{Conclusion}

Our formulation of decision problems has several advantages over more traditional formulations. First, we theorize about only the actual observable choices available to the DM, without having states and outcomes, and without needing to view choices as functions from states to outcomes. In contrast, in many decision theory experiments, when the DM is given a word problem, the experimenter has an interpretation of this problem as a choice among Savage acts. The experimenter is then really testing whether the DM's choices are consistent with decision theory given this interpretation.

Thus, a joint hypothesis is being tested. Standard decision theory can be rejected only if the other part of the joint test-that the experimenter and DM interpret 
represent the word problem with identical Savage acts-is maintained as true. As we have shown, given the decision maker's theory, our approach places restrictions on choices. Thus, although our approach also leads to a joint hypothesis, we have a framework for reasoning about what the decision maker knows about the world (a theory) and about what restrictions on choices arise from SEU given the decision maker's theory, rather than the experimenter's theory. In fact, if an experimenter is willing to take the existence of an SEU representation as a maintained hypothesis, she can test hypotheses about the DMs theory of the world.

Second, by viewing choices as syntactic objects, our approach allows us to consider DMs who associate different meanings to the same object of choice. Moreover, that meaning can depend on the DM's theory of the world. A DM might have a theory that does not recognize equivalences between certain tests, and thus choices, that may be obvious to others. This potential difference between a DM's theory of the world and an experimenter's view of the world provides an explanation for framing effects, while still allowing us to view a DM as an expected utility maximizer. Moreover, since a DM's theory may not contain all of standard propositional logic, we can model resource-bounded DMs who cannot discern all the logical consequences of their choices. The existence of an SEU representation and the presence of framing effects are independent once one is free to choose a state space.

Third, our approach allows us to consider different DMs who use different languages to describe the same phenomena. To see why this might be important, consider two decision makers who are interested in 100 shares of Alphabet stock and money (as in Example 10). Suppose that one DM considers quantitative issues like the price/earnings ratio to be relevant to the future value of Alphabet, while the other considers astrological tables relevant to Alphabet's future value. The DM who uses astrology might not understand price/earnings ratios (the notion is simply not in his vocabulary) and, similarly, the DM who uses quantitative methods might not understand what it means for the moon to be in the seventh house. Nevertheless, they can trade Alphabet stock and money, as long as they both have available primitive actions like 'buy 100 shares of Alphabet' and 'sell 100 shares of Alphabet'. If we model these decision problems in the Savage framework, we would have to think of assets as Savage acts on a common state and outcome space. Our approach does not require us to tie the DM's decision problems together with a common state space. Every DM acts as if she has a state space, but these state spaces may be different. Even if agents agree on the formulas of interest, they may interpret them completely differently. Thus, agreeing to disagree results (Aumann, 1976), which say that DMs 
with a common prior must have a common posterior (they cannot agree to disagree) will in general not hold.

A fourth advantage of our approach is more subtle, but potentially profound. Representation theorems are just that; they merely provide a description of a preference relation in terms of numerical scales. Decision theorists make no pretense that these representations have anything to do with the cognitive processes by which individuals make decisions. But to the extent that the language of choices models the language of the DM, we have the ability to interpret the effects of cognitive limitations having to do with the language in terms of the representation. Our approach allows us to consider the possibility that there may be limitations on the space of choices because some sequence of tests is too computationally costly to verify. Our model of nonstandard test interpretations also takes into account a DM's potential inability to recognize that two choices logically represent the same function.

There is clearly more than could be done to develop our approach and apply it in various settings. One obvious development would be to consider decision making over time, which will require us to consider learning in more detail. Learning in our framework is not just a matter of conditioning, but also learning about new notions (i.e., becoming aware of new tests). Note that considering dynamic decisionmaking will require us to take a richer collection of objects of choice, a programming language that allows (among other things) sequential actions (do this, then do that, then do that). A second direction to consider is multi-agent decision making. As we have suggested in examples, once we move to a multi-agent case, we can consider agents who may use different languages. There is clearly a connection here between our framework and the burgeoning literature on awareness and its applications to game theory (see, for example, (Feinbera, 2004, Fagin and Halpern, 1988, Halpern, 2001, Halpern and Rêgo, 2006, Heifetz et all, 2006, Karni and Vierø, 2013, Modica and Rustichini, 1999) that needs to be explored.

We have shown how our approach can model framing problems as a consequence of the agent having a different theory from the modeler. We believe that our approach can also model other 'deviations' from rationality of the type reported by Luce (1990), such as failures of particular accounting identities that lead to the requirement that a DM is indifferent between formally equivalent gambles. These deviations can be viewed as a consequence of an agent's bounded processing power. This will require us to be able to distinguish sentences such as, for example, $2 / 3(1 / 4 a+$ $3 / 4 b)+1 / 3 c$ and $1 / 6 a+5 / 6(3 / 5 b+2 / 5 c)$. To do that, we need to give seman- 
tics to choices that does not view them as functions from states to distributions over outcomes. We leave further exploration of all these issues to future work.

\section{Appendix}

Proof of Proposition 1. First suppose that cancellation holds. To see that $\succeq$ is reflexive, take $n=1$ and $a_{1}=b_{1}=a$ in the cancellation axiom. The hypothesis of the cancellation axiom clearly holds, so we must have $a \succeq a$. To see that cancellation implies transitivity, consider the pair of sequences $\langle a, b, c\rangle$ and $\langle b, c, a\rangle$. Cancellation clearly applies. If $a \succeq b$ and $b \succeq c$, then cancellation implies $a \succeq c$.

We also need to prove the converse part of the proposition. For the converse, suppose that $\succeq$ is reflexive and transitive. By way of contradiction, suppose that $\left\langle a_{1}, \ldots, a_{n}\right\rangle$ and $\left\langle b_{1}, \ldots, b_{n}\right\rangle$ are two sequences of minimal cardinality $n$ that violate cancellation; that is, $\left\{\left\{a_{1}, \ldots, a_{n}\right\}\right\}=\left\{\left\{b_{1}, \ldots, b_{n}\right\}\right\}, a_{i} \succeq b_{i}$ for $i \in\{1, \ldots, n-1\}$, and it is not the case that $b_{n} \succeq a_{n}$

If $n=1$, and $\{\{a\}\}=\{\{b\}\}$, then we must have $a=b$, and the cancellation postulate holds iff $a \succeq a$, which follows from our assumption that $\succeq$ is reflexive.

If $n>1$, since the two multisets are equal, there must be some permutation $\tau$ of $\{1, \ldots, n\}$ such that $a_{\tau(i)}=b_{i}$. Let $\tau^{j}(1)$ be the result of applying $\tau j$ times, beginning with 1 . Let $k$ be the first integer such that $\tau^{k+1}(1)$ is either 1 or $n$. Then we have the situation described by the following table, where the diagonal arrow denotes equality.

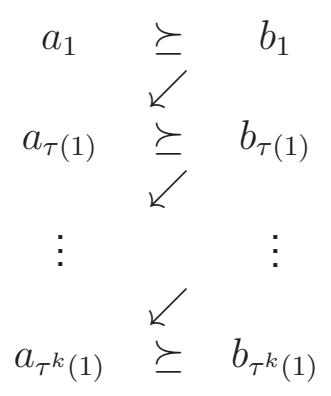


Note that we must have $k \leq n-1$. If $\tau^{k+1}(1)=1$, then $b_{\tau^{k}(i)}=a_{1}$. Thus, the multisets $\left\{\left\{a_{1}, \ldots, a_{\tau^{k}(1)}\right\}\right\}$ and $\left\{\left\{b_{1}, \ldots, b_{\tau^{k}(1)}\right\}\right\}$ must be equal. The sequences that remain after removing $\left\{\left\{a_{1}, \ldots, a_{\tau^{k}(1)}\right\}\right\}$ from the first sequence and $\left\{\left\{b_{1}, \ldots, b_{\tau^{k}(1)}\right\}\right\}$ from the second also provide a counterexample to the cancellation axiom, contradicting the minimality of $n$. Thus, $\tau^{k+1}(1)=n$, and we can conclude by transitivity that $a_{1} \succeq a_{n}$.

Continuing on with the iteration procedure starting with $a_{\tau^{k+2}(1)}=a_{\tau(n)}=b_{n}$, we ultimately must return to $a_{1}$ and $b_{1}$, as illustrated in the following table: $a_{1}$ and $b_{1}$.

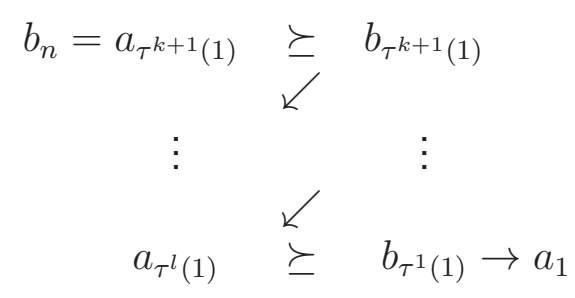

It follows from transitivity that $b_{n} \succeq a_{1}$. By another application of transitivity, we conclude that $b_{n} \succeq a_{n}$. This contradicts the hypothesis that the original sequence violated the cancellation axiom.

Proof of Proposition 2. Let $P O(C)$ denote all the preference relations $\succeq$ on $C$. Since $C$ is finite, so is $P O(C)$. Let $P O(C, n)$ denote the subset of $P O(C)$ consisting of all preference relations $\succeq$ satisfying $S C_{1}, \ldots, S C_{n} . P O(C, 1), P O(C, 2), \ldots$ is clearly a nondecreasing sequence of sets of preference relations. Since $P O(C)$ is finite, the sequence must stabilize at some point; that is, there must exist some $N$ such that $P O(C, n)=P O(C, N)$ for all $n \geq N$. It follows that if a preference relation $\succeq$ on $C$ satisfies $S C_{1}, \ldots, S C_{N}$, then it satisfies $S C_{n}$ for all $n$.

Proof of Proposition 3. Take $\left\langle a_{1}, a_{2}\right\rangle=\left\langle a_{T} c, b_{T} c^{\prime}\right\rangle$ and take $\left\langle b_{1}, b_{2}\right\rangle=\left\langle b_{T} c, a_{T} c^{\prime}\right\rangle$. Note that for each state $s \in T,\left\{\left\{a_{T} c(s), b_{T} c^{\prime}(s)\right\}\right\}=\{\{a(s), b(s)\}\}=$ $\left\{\left\{b_{T} c(s), a_{T} c^{\prime}(s)\right\}\right\}$, and for each state $s \notin T,\left\{\left\{a_{T} c(s), b_{T} c^{\prime}(s)\right\}\right\}=$ $\left\{\left\{c(s), c^{\prime}(s)\right\}\right\}=\left\{\left\{b_{T} c(s), a_{T} c^{\prime}(s)\right\}\right\}$. Thus, we can apply statewise cancellation to get that if $a_{T} c \succeq b_{T} c$, then $a_{T} c^{\prime} \succeq b_{T} c^{\prime}$.

Proof of Proposition 4. Suppose the hypotheses of extended statewise cancellation hold. If $b_{n} \succeq a_{n}$, we are done. If not, by $\mathbf{A} 1, a_{n} \succeq b_{n}$. But then the hypotheses of statewise cancellation hold, so again, $b_{n} \succeq a_{n}$. 
Proof of Theorem 1. Suppose that $\succeq$ satisfies extended mixture cancellation. Then it satisfies cancellation, and so from Proposition $1, \succeq$ is reflexive and transitive. To show that $\succeq$ satisfies rational mixture independence, suppose that $a \succeq b$ and $r=$ $m / n$. Let $a_{1}=\cdots=a_{m}=a$ and $a_{m+1}=\cdots=a_{m+n}=r b+(1-r) c$; let $b_{1}=$ $\cdots=b_{m}=b$ and $b_{m+1}=\cdots=b_{m+n}=r a+(1-r) c$. Then $\sum_{i=1}^{m+n} a_{i}=\sum_{i=1}^{m+n} b_{i}$, and so $r a+(1-r) c \succeq r b+(1-r) c$.

Similarly, if $r a+(1-r) c \succeq r b+(1-r) c$, then applying extended mixture cancellation to the same sequence of acts shows that $a \succeq b$.

For the converse, suppose that $\succeq$ is reflexive, transitive, and satisfies rational mixture independence. Suppose that $\left\langle a_{1}, \ldots, a_{n}\right\rangle$ and $\left\langle b_{1}, \ldots, b_{n}\right\rangle$ are sequences of of elements of $C$ such that $a_{1}+\cdots+a_{n}=b_{1}+\cdots+b_{n}, a_{i} \succeq b_{i}$ for $i=1, \ldots, n-k$, $a_{k+1}=\ldots=a_{n}$, and $b_{k+1}=\ldots=b_{n}$. Then from transitivity and rational mixture independence we get that

$$
\begin{aligned}
\frac{1}{n}\left(a_{1}+\cdots+a_{n}\right) \succeq \frac{1}{n}\left(b_{1}+\cdots+b_{k}+a_{k+1}+\right. & \left.\cdots+a_{n}\right) \\
& =\frac{1}{n}\left(b_{1}+\cdots+b_{k}\right)+\frac{n-k}{n} a_{n} .
\end{aligned}
$$

Since $b_{k+1}=\ldots=b_{n}$ and $a_{1}+\cdots+a_{n}=b_{1}+\cdots+b_{n}$, we have that

$$
\frac{1}{n}\left(b_{1}+\cdots+b_{k}\right)+\frac{n-k}{n}\left(b_{n}\right)=\frac{1}{n}\left(b_{1}+\cdots+b_{n}\right)=\frac{1}{n}\left(a_{1}+\cdots+a_{n}\right) .
$$

Thus, by transitivity,

$$
\frac{1}{n}\left(b_{1}+\cdots+b_{k}\right)+\frac{n-k}{n}\left(b_{n}\right) \succeq \frac{1}{n}\left(b_{1}+\cdots+b_{k}\right)+\frac{n-k}{n}\left(a_{n}\right) .
$$

By rational mixture independence, it follows that $b_{n} \succeq a_{n}$.

Proof of Proposition 5. Let $S=\operatorname{At}_{\mathcal{T}}\left(T_{0}\right)$, the set of atoms consistent with $\mathcal{T}$, let $O$ be $\mathcal{A}_{0}$, the set of primitive choices, and define $\rho_{S O}^{0}(c)$ to be the constant function $c$ for a primitive choice $c$. It is easy to see that $\rho_{S O}(c)=f_{c}$ for all choices $c$. If $a \equiv \mathcal{T} b$, then $\rho_{S O}(a)=\rho_{S O}(b)$, so we must have $f_{a}=f_{b}$. Now apply A2 with $a_{1}=a$ and $b_{1}=b$ to get $b \succeq a$, and then reverse the roles of $a$ and $b$.

Proof of Proposition 6. For part (a), suppose that $a \succeq b$, and $c$ is an arbitrary act. By Theorem 1, rational mixture independence holds, so we have $r a+(1-r) c \succeq$ 
$r b+(1-r) c$ for all rational $r$. By A3, we have $r a+(1-r) c \succeq r b+(1-r) c$ for all real $r$. Conversely, suppose that $r a+(1-r) c \succeq r b+(1-r) c$ for some real $r$. If $r$ is rational, it is immediate from rational mixture independence that $a \succeq b$. If $r$ is not rational, choose a rational $r^{\prime}$ such that $0<r^{\prime}<r$. Then we can find a sequence of rational numbers $r_{n}$ such that $r_{n} r$ converges to $r^{\prime}$. By rational mixture independence, $r_{n}(r a+(1-r) c)+\left(1-r_{n}\right) c \succeq r_{n}(r b+(1-r) c)+\left(1-r_{n}\right) c$. By A3, it follows that $r^{\prime} a+\left(1-r^{\prime}\right) c \succeq r^{\prime} b+\left(1-r^{\prime}\right) c$. Now by rational mixture independence, we have $a \succeq b$, as desired.

For part (b), suppose that $a \succ b \succ c$. Mixture independence (which follows from $\mathbf{A} 2^{\dagger}$ and $\mathbf{A} 3$, as we have observed) implies that, for all $r \in(0,1), a \succ r a+$ $(1-r) c$. To see that $r a+(1-r) c \succ b$ for some $r \in(0,1)$, suppose not. Then, by $\mathbf{A} 1, b \succeq r a+(1-r) c$ for all $r \in(0,1)$, and by $\mathbf{A} 3$, we have that $b \succeq a$, contradicting our initial assumption. The remaining inequalities follow in a similar fashion.

We now prove the representation theorems: Theorems 2, 3, and 4, They all use essentially the same technique. It is convenient to start with Theorem 3 . The first step is to get an additively separable utility representation for AA acts on a state space $S$ with outcome space $O$. This result is presented in Theorem 5 which is somewhat novel because we use extended mixture cancellation and $\mathbf{A} \mathbf{3}$ rather than independence and Arch, and because $\succeq$ can be incomplete.

Theorem 5. A preference relation $\succeq$ on a set $C$ of mixture-closed $A A$ acts mapping a finite set $S$ of states to distributions over a finite set $O$ of outcomes satisfies Extended Mixture Cancellation and $\mathbf{A} 3$ iff there exists a set $\mathcal{U}$ of utility functions on $S \times O$ such that $a \succeq b$ iff

$$
\sum_{s \in S} \sum_{o \in O} u(s, o) a(s)(o) \geq \sum_{s \in S} \sum_{o \in O} u(s, o) b(s)(o)
$$

for all $u \in \mathcal{U}$. Moreover, $\succeq$ also satisfies $\boldsymbol{A} 1$ iff we can take $\mathcal{U}$ to be a singleton $\{u\}$. In this case, $u$ is unique up affine transformations: if $u^{\prime}$ also satisfies (3), then there exist $\alpha$ and $\beta$ such that $u^{\prime}=\alpha u+\beta$.

Proof. In the totally ordered case, this result is well known. Indeed, for a preference relation $\succeq$ that satisfies A1, Proposition 7.4 of (Kreps, 1988) shows that such a representation holds iff $\succeq$ satisfies Arch, mixture independence, transitivity, and reflexivity. Theorem 1 and Proposition 6 show that if $\succeq$ satisfies extended mixture cancellation, A1, and A3, then these properties hold, so there is a representation. 
Conversely, if there is such a representation, then all these properties are easily seen to hold. It follows that in the presence of A1, extended mixture cancellation and A3 are equivalent to these properties. However, since we do not want to assume A1, we must work a little harder. Fortunately, the techniques we use will be useful for our later results.

To see that the existence of a representation implies Extended Mixture Cancellation and A3, first consider Extended Mixture Cancellation, and suppose that $\left\langle a_{1}, \ldots, a_{n}\right\rangle$ and $\left\langle b_{1} \ldots, b_{n}\right\rangle$ are such that $a_{1} \succeq b_{1}, \ldots, a_{k} \succeq b_{k}, a_{k+1}=\ldots=a_{n}$, $b_{k+1}=\cdots=b_{n}$, and $a_{1}+\cdots+a_{n}=b_{1}+\cdots+b_{n}$. For all $u \in \mathcal{U}$, for $i=1, \ldots, k$, we have

$$
\sum_{s \in S} u\left(s, a_{i}(s)\right) \geq \sum_{s \in S} u\left(s, b_{i}(s)\right) .
$$

Since $a_{1}+\cdots+a_{n}=b_{1}+\cdots+b_{n}$, for all $s \in S$, it must be that, for all $u \in \mathcal{U}$,

$$
\sum_{i=1}^{n} \sum_{s \in S} u\left(s, a_{i}(s)\right)=\sum_{i=1}^{n} \sum_{s \in S} u\left(s, b_{i}(s)\right) .
$$

Thus, for all $u \in \mathcal{U}$,

$$
\sum_{i=k+1}^{n} \sum_{s \in S} u\left(s, a_{i}(s)\right) \leq \sum_{i=k+1}^{n} \sum_{s \in S} u\left(s, b_{i}(s)\right) .
$$

Since $a_{k+1}=\ldots=a_{n}$ and $b_{k+1}=\ldots=b_{n}$, it easily follows that, for all $u \in U$,

$$
\sum_{s \in S} u\left(s, a_{n}(s)\right) \leq \sum_{s \in S} u\left(s, b_{n}(s)\right) .
$$

Thus $b_{n} \succeq a_{n}$, as desired. The fact that $\mathbf{A} \mathbf{3}$ holds is straightforward, and left to the reader.

For the 'if' direction, recall that we can view the elements of $C$ as vectors in $\mathbf{R}^{|\mathbf{S}| \times|\mathbf{O}|}$. For the rest of this proof, we identify elements of $C$ with such vectors. Let $D=\{a-b: a, b \in C\}$, and let $D^{+}=\{a-b: a \succeq b\}$. Recall that a (pointed) cone in $\mathbf{R}^{|\mathbf{S}| \times|\mathbf{O}|}$ is a set $C C$ that is closed under nonnegative linear combinations, so that if $c_{1}, c_{2} \in C C$ and $\alpha, \beta \geq 0$, then $\alpha c_{1}+\beta c_{2} \in C C$. We need the following lemma.

Lemma 1. There exists a closed convex cone $C C$ such that $D^{+}=C C \cap D$. 
Proof. Let $C C$ consist of all vectors of the form $\alpha_{1} d_{1}+\cdots+\alpha_{n} d_{n}$ for some $n>$ 0 , where $d_{1}, \ldots, d_{n} \in D^{+}$and $\alpha_{1}, \ldots, \alpha_{n} \geq 0$. Clearly $C C$ is a convex cone, and closed because $D^{+}$is finite. Also, $D^{+} \subseteq C C \cap D$. For the opposite inclusion, suppose that $\alpha_{1} d_{1}+\cdots+\alpha_{n} d_{n}=d$, where $d_{1}, \ldots, d_{n} \in D^{+}, d \in D$, and $\alpha_{1}, \ldots, \alpha_{n} \geq 0$. Thus, there must exist $a_{1}, \ldots, a_{n}, b_{1}, \ldots, b_{n}, a, b \in C$ such that $a-b=d, a_{i}-b_{i}=d_{i}$, and $a_{i} \succeq b_{i}$ for $i=1, \ldots, n$. We want to show that $d \in D^{+}$ or, equivalently, that $a \succeq b$. Let $r=\alpha_{1}+\cdots+\alpha_{n}+1$. Since $C$ is mixture-closed, both $\left(\alpha_{1} / r\right) a_{1}+\cdots+\left(\alpha_{n} / r\right) a_{n}+(1 / r) b \in C$ and $\left(\alpha_{1} / r\right) b_{1}+\cdots+\left(\alpha_{n} / r\right) b_{n}+(1 / r) a$ are in $C$. Moreover, since $\alpha_{1} d_{1}+\cdots+\alpha_{n} d_{n}=d$ and $a_{i} \succeq b_{i}$ for $i=1, \ldots, n$, it easily follows from mixture independence (which is a consequence of extended mixture cancellation) that $\left(\alpha_{1} / r\right) b_{1}+\cdots+\left(\alpha_{n} / r\right) b_{n}+(1 / r) a=\left(\alpha_{1} / r\right) a_{1}+\cdots+\left(\alpha_{n} / r\right) a_{n}+(1 / r) b \succeq$ $(1 / r) b_{1}+\cdots+(1 / r) b_{n}+(1 / r) b$. Another application of mixture independence gives us $a \succeq b$, as desired.

Returning to the proof of Theorem 5, note that if $a \succeq b$ for all $a$ and $b$, then $D^{+}=D$, and we can take $C C$ to be the whole space. If $\succeq$ is nontrivial, then $C C$ is not the whole space. It is well known (Rockafellar, 1970) that every closed cone that is not the whole space is the intersection of closed half-spaces (where a half-space is characterized by a vector $u$ such and consists of all the vectors $x$ such that $u \cdot x \geq 0$ ). Given our identification of elements of $C$ with vectors, we can identify the vector $u$ in $\mathbf{R}^{|\mathbf{S}| \times|\mathbf{O}|}$ characterizing a half-space with a (state-dependent) utility function, where $u(s, o)$ is the $(s, o)$ component of the vector $u$. If $C C$ is the whole space, we can get a representation by simply taking $\mathcal{U}$ to consist of the single utility function such that $u(s, o)=0$ for all $(s, o) \in S \times O$. Otherwise, we can take $\mathcal{U}$ to consist of the utility functions characterizing the half-spaces containing $C C$. It is easy to see that for $a, b \in C$, we have that $a \succeq b$ iff $a-b \in D^{+}$iff $a-b \in C C$ iff $u \cdot(a-b) \geq 0$ for every half-space $u$ containing $C C$; i.e. iff (3) holds.

To prove Theorem 3 , the following lemma, which shows that we can identify complete preference relations with half-spaces, is also useful. Given a subset $R$ of $\mathbf{R}^{|\mathbf{S}| \times|\mathbf{O}|}$, define the relation $\succeq_{R}$ on $C$ by taking $a \succeq_{R} b$ iff $a-b \in R$.

Lemma 2. $E X_{\mathrm{AX}}=\left\{\succeq_{R}: R\right.$ is either a half-space containing $C C$ or the full space $\}$.

Proof. If $R$ is the full space, then $\succeq_{R}$ is the trivial relation, so clearly $\succeq_{R} \in E X_{\mathrm{AX}}(\succeq)$. If $R$ is a half-space $H$ containing $C C$ and $H$ is characterized by $u$, then $\succeq_{H}$ extends $\succeq$, since $C C \subseteq H$. To see that $\succeq_{H}$ satisfies $\mathbf{A} 1$, observe that if $(a, b) \notin \succeq_{H}$, then 
$u \cdot(a-b)<0$, so $u \cdot(b-a)>0$, and $b \succeq_{H} a$. To see that $\succeq_{H}$ satisfies $\mathbf{A} 2^{\prime}$, suppose that $a_{1}+\cdot+a_{n}=b_{1}+\cdot+b_{n}$, and $a_{i} \succeq_{H} b_{i}$ for $i=1, \ldots, n-1$. Thus, $u \cdot\left(a_{1}+\cdot+a_{n}\right)=u \cdot\left(b_{1}+\cdot b_{n}\right)$, and $u \cdot\left(a_{i}-b_{i}\right) \geq 0$ for $i=1, \ldots, n-1$. It follows that $\left(b_{n}-a_{n}\right) \cdot u \geq 0$, so $b_{n} \succeq_{H} a_{n}$. Finally, for $\mathbf{A 3}$, it is clear that if $\left(a_{n}, b_{n}\right) \rightarrow(a, b)$, and $u \cdot\left(a_{n}-b_{n}\right) \geq 0$, then $u \cdot(a-b) \geq 0$, so $a \succeq_{H} b$. Thus, $\succeq_{H} \in E X_{\mathrm{AX}}(\succeq)$.

For the opposite inclusion, suppose that $\succeq^{\prime} \in E X_{\mathrm{AX}}(\succeq)$. Let $C C^{\prime}$ be the cone determined by $\succeq^{\prime}$, as in Lemma1. Clearly $C C \subseteq C C^{\prime}$. If $C C^{\prime}$ is the full space, then we are done, since $\succeq^{\prime}=\succeq_{C C^{\prime}}$. Otherwise, $C C^{\prime}$ is the intersection of half-spaces. Choose a half-space $H$ such that $C C^{\prime} \subseteq H$. We claim that $\succeq^{\prime}=\succeq_{H}$. Suppose not. Since $C C^{\prime} \subseteq H$, we must have $\succeq^{\prime} \subseteq \succeq_{H}$. There must exist $a, b \in C$ such that $a \succeq_{H} b$ and $a \succeq^{\prime} b$. Since $\succeq^{\prime}$ is complete, we must have $b \succ^{\prime} a$. Thus, $b \succeq_{H} a$, so $a \sim_{H} b$. Since $H$ is not the full space, there must be some $c$ such that $b \chi_{H} c$. Suppose that $c \succ_{H} b$. We must have $c \succ b$, since otherwise $b \succeq^{\prime} c$, and it follows that $b \sim_{H} c$. By the Archimedean property (which holds by Proposition 6), since $c \succ^{\prime} b \succ^{\prime} a$, there exists $r>0$ such that $b \succ^{\prime} r c+(1-r) a$. Thus we must have $b \succeq_{H} r c+(1-r) a \succeq_{H} b$. But this contradicts the assumption that $c \succ_{H} b \sim_{H} a$. We get a similar contradiction if $b \succ_{H} c$, since then $a \succ_{H} c$.

Proof of Theorems 3 and $3 c$. It is easy to check that if there is a constructive SEU representation of $\succeq$, then $\succeq$ satisfies $\mathrm{A} 2^{\dagger}$ and A3. For the converse, suppose that $\succeq$ satisfies $\mathbf{A} 2^{\dagger}$ and A3. Take $S=\operatorname{At}_{\mathcal{T}}\left(T_{0}\right)$ and $O^{\prime}=\mathcal{A}_{0}$. Define $\pi_{S}(t)$ to be the set of all atoms $\delta$ in $\operatorname{At}_{\mathcal{T}}\left(T_{0}\right)$ such that $\delta \Rightarrow t$. Define $\rho_{S O^{\prime}}^{0}(a)$ to be the constant function $a$ for a primitive choice $a$. It is easy to see that $\rho_{S O^{\prime}}(a)=f_{a}$ for all choices $a \in C$. Define a preference relation $\succeq_{S}$ on the AA acts of the form $\rho_{S O^{\prime}}(a)$ by taking $f_{a} \succeq_{S} f_{b}$ iff $a \succeq b$. The fact that $\succeq_{S}$ is well defined follows from Proposition [5, for if $f_{a}=f_{a^{\prime}}$, then it easily follows that $a \equiv_{\mathcal{T}} a^{\prime}$, so $a \sim a^{\prime}$. Clearly $\succeq_{S}$ satisfies extended statewise cancellation, and satisfies A1 iff $\succeq$ does. Thus, by Theorem [5, there is an additively separable representation of $\succeq$.

Now we adjust the state and outcome spaces to get a constructive SEU representation. Suppose first that $\mathbf{A} 1$ holds. Take $S=\operatorname{At}_{\mathcal{T}}\left(T_{0}\right)$ and take $O=$ $\operatorname{At}_{\mathcal{T}}\left(T_{0}\right) \times \mathcal{A}_{0}$. For a primitive choice $a \in \mathcal{A}_{0}$, define $\rho_{S O}^{0}(a)(\delta)=(\delta, a)$. To complete the proof, it clearly suffices to find a probability measure $p$ on $\operatorname{At}_{\mathcal{T}}\left(T_{0}\right)$ and a utility function $v$ on $\operatorname{At}_{\mathcal{T}}\left(T_{0}\right) \times \mathcal{A}_{0}$ such that $u(\delta, a)=p(\delta) v(\delta, a)$, where $u$ is the state-dependent utility function whose existence is guaranteed by Theorem [5. This is accomplished by taking any probability measure $p$ on $\operatorname{At}_{\mathcal{T}}\left(T_{0}\right)$ such that for all atoms $\delta, p(\delta)>0$, and taking $v(\delta, a)=u(\delta, a) / p(\delta)$. 
If $\mathbf{A} \mathbf{1}$ does not hold, then proceed as above, using Theorem [5] to get an entire set $\mathcal{U}^{\prime}$ of functions $u: \operatorname{At}_{\mathcal{T}}\left(T_{0}\right) \times \mathcal{A}_{0} \rightarrow \mathbf{R}$, and a single probability distribution $p$ that assigns positive probability to every atom. Let $\mathcal{U}$ consist of all utility functions $u$ such that there exists some $u^{\prime} \in \mathcal{U}^{\prime}$ such that $u(\alpha, \delta)=u^{\prime}(\delta, a) / p(\delta)$. In this representation, again, the state space is $\operatorname{At}_{\mathcal{T}}\left(T_{0}\right)$.

We now give a representation using a single utility function. Let $S^{\prime}=\operatorname{At}_{\mathcal{T}}\left(T_{0}\right) \times$ $E X_{\mathrm{AX}}(\succeq)$ and let $O^{\prime \prime}=\operatorname{At}_{\mathcal{T}}\left(T_{0}\right) \times \mathcal{A}_{0} \times E X_{\mathrm{AX}}(\succeq)$. Define $\rho_{S^{\prime} O^{\prime \prime}}^{0}(a)\left(\delta, \succeq^{\prime}\right)=\left(\delta, a, \succeq^{\prime}\right)$. For $t \in T_{0}$, define $\pi_{S^{\prime}}(t)=\pi_{S}(t) \times E X_{\mathrm{AX}}(\succeq)$. As before, let $\mathcal{U}^{\prime}$ be the set of utility functions on $\operatorname{At}_{\mathcal{T}}\left(T_{0}\right) \times \mathcal{A}_{0}$ that represent $\succeq$. Lemma 2 shows that $\mathcal{U}^{\prime}$ consists of one utility function $u_{\succeq^{\prime}}$ for every total order $\succeq^{\prime} \in E X_{\mathrm{AX}}(\succeq)$. Again, fix a probability measure $p$ on $\operatorname{At}_{\mathcal{T}}\left(\bar{T}_{0}\right)$ such that $p(\delta)>0$ for all $\delta \in \operatorname{At}_{\mathcal{T}}\left(T_{0}\right)$. For each relation $\succeq^{\prime} \in E X_{\mathrm{AX}}(\succeq)$, define $p_{\succeq^{\prime}}$ on $\operatorname{At}_{\mathcal{T}}\left(T_{0}\right) \times E X_{\mathrm{AX}}(\succeq)$ by taking $p_{\succeq^{\prime}}\left(\delta, \succeq^{\prime \prime}\right)=p(\delta)$ if $\succeq^{\prime}=\succeq^{\prime \prime}$, and $p_{\succeq^{\prime}}\left(\delta, \succeq^{\prime \prime}\right)=0$ if $\succeq^{\prime} \neq \succeq^{\prime \prime}$. Let $\mathcal{P}=\left\{p_{\succeq^{\prime}}: \succeq^{\prime} \in E X_{\mathrm{AX}}(\succeq)\right\}$. Define $v\left(\delta, a, \succeq^{\prime}\right)=u_{\succeq^{\prime}}(\delta, a) / p(\delta)$. It is easy to see that $\mathcal{P} \times\{v\}$ represents $\succeq$. Moreover, it easily follows that, with this representation, $\succeq_{t}$ and $\succeq \oplus(a, b)$ can be represented by updating.

Now we show how the ideas in this proof can be modified to prove Theorems 2 and 2 r.

Proof of Theorems 2 and $2 \mathrm{c}$. Again, it is easy to check that if there is a constructive SEU representation of $\succeq$, then $\succeq$ satisfies $\mathrm{A} 2^{\prime}$.

For the converse, suppose that we are given a preference relation $\succeq$ that satisfies $\mathrm{A} 2^{\prime}$. The structure of the proof is identical to that of Theorem 3 . We first prove an analogue of Theorem 5 .

Theorem 6. A preference relation $\succeq$ on a set $C$ of Savage acts mapping a finite set $S$ of states to a finite set $O$ of outcomes satisfies extended statewise cancellation iff there exists a set $\mathcal{U}$ of utility functions on $S \times O$ such that $a \succeq b$ iff

$$
\sum_{s \in S} u(s, a(s)) \geq \sum_{s \in S} u(s, b(s)) \text { for all } u \in \mathcal{U} \text {. }
$$

Moreover, $\succeq$ satisfies $\mathbf{A 1}$ iff $\mathcal{U}$ can be chosen to be a singleton 5

\footnotetext{
${ }^{5}$ This result is a generalization of Theorem 4.1 of Fishburn (1970), which can be viewed as considering the case where $|S|=1$ and the preference relation $\succeq$ is complete (i.e., satisfies A1).
} 
The proof of Theorem 6 is identical to that of Theorem [5] except that we need an analogue of Lemma 1 for the case that $\succeq$ satisfies $A 2^{\prime}$. Let $D$ and $D^{+}$be defined as in Lemma1.

Lemma 3. If $\succeq$ satisfies $\mathbf{A} 2^{\prime}$, there exists a cone $C C$ such that $D^{+}=C C \cap D$.

Proof. Again, let $C C$ consist of all vectors of the form $\alpha_{1} d_{1}+\cdots+\alpha_{n} d_{n}$ for some $n>0$, where $d_{1}, \ldots, d_{n} \in D^{+}$and $\alpha_{1}, \ldots, \alpha_{n} \geq 0$. Clearly $C C$ is a cone. Since $C$ is closed and bounded, so is $D$, and $\mathbf{A} 3$ implies that $D^{+}$is closed and bounded. Therefore $C C$ is closed. Furthermore, $D^{+} \subseteq C C \cap D$. For the converse inclusion, suppose that $\alpha_{1} d_{1}+\cdots+\alpha_{n} d_{n}=d$, where $d_{1}, \ldots, d_{n} \in D^{+}, d \in D$, and $\alpha_{1}, \ldots, \alpha_{n} \geq 0$. That means that $\alpha_{1}, \ldots, \alpha_{n}$ is a nonnegative solution to the system of equations $x_{1} d_{1}+\cdots+x_{n} d_{n}=d$. Since all the coefficients in these equations are rational (in fact, they are all 0,1 , and -1 ) there exists a nonnegative rational solution to this system of equations. It easily follows that there exist positive integers $\beta_{1}, \ldots, \beta_{n+1}$ such that $\beta_{1} d_{1}+\cdots+\beta_{n} d_{n}=\beta_{n+1} d$. By definition, there must exist $a_{1}, \ldots, a_{n}, b_{1}, \ldots, b_{n}, a, b \in C$ such that $a-b=d, a_{i}-b_{i}=d_{i}$, and $a_{i} \succeq b_{i}$ for $i=1, \ldots, n$. It follows that $\left\{\left\{a_{1}, \ldots, a_{1}, \ldots, a_{n}, \ldots, a_{n}, b, \ldots, b\right\}\right\}=$ $\left\{\left\{b_{1}, \ldots, b_{1}, \ldots, a_{n}, \ldots, a_{n}, b, \ldots, b\right\}\right\}$, where $a_{i}$ occurs in the left-hand multiset $\beta_{i}$ times and $b$ occurs $\beta_{n+1}$ times, and, similarly, $b_{i}$ occurs in the right-hand side $\beta_{i}$ times and $a$ occurs $\beta_{n+1}$ times. A2 ${ }^{\prime}$ now implies that $a \succeq b$, so $d \in C C$, as desired.

The proof of Theorem 6 is now identical to that of Theorem 5. Moreover, the proof of Theorem 2 now follows from Theorem 6 in exactly the same way that the proof of Theorem 3 follows from Theorem [5.

Proof of Theorems 4 and $4 \mathbf{c}$. First, let $S=\operatorname{At}_{\mathcal{T}}\left(T_{0}\right)$ and $O^{\prime}=\mathcal{A}_{0}$. As in the proof of Theorem 3 , using Theorem [5, we can find an additively separable representation of $\succeq$; that is, we can find a set $\mathcal{U}$ of utility functions on $S=\operatorname{At}_{\mathcal{T}}\left(T_{0}\right) \times \mathcal{A}_{0}$ that represent $\succeq$. Let $o_{1}$ and $o_{0}$ denote the best and worst outcomes guaranteed to exist by A4. Note that it follows from $\mathbf{A} 4$ and $\mathbf{A} 5$ that $u\left(\delta, o_{0}\right) \leq u(\delta, a) \leq u\left(\delta, o_{1}\right)$ for all $(\delta, a) \in$ $S$. (For null atoms $\delta$, we must in fact have $u\left(\delta, o_{0}\right)=u(\delta, a)=u\left(\delta, o_{1}\right)$ for all $a \in \mathcal{A}_{0}$ ). Furthermore, note that we can replace $u$ by $u^{\prime}$, where $u^{\prime}(\delta, a)=u(\delta, a)-u\left(\delta, o_{0}\right)$ for all $(\delta, a) \in S$ to get an equivalent representation; thus, by appropriate scaling, we can assume without loss of generality that, for all $u \in \mathcal{U}$, we have that $u\left(\delta, o_{0}\right)=0$ for all $\delta \in \operatorname{At}_{\mathcal{T}}\left(T_{0}\right)$ (so $u(\delta, a) \geq 0$ for all $(\delta, a) \in S$ ) and that $\sum_{\delta^{\prime} \in \operatorname{At}_{\mathcal{T}}\left(T_{0}\right)} u_{\succeq^{\prime}}\left(\delta^{\prime}, o_{1}\right)=1$. Finally, note that it easily follows from A3 that, for all $o \in O$, there exists a unique 
$c_{o} \in[0,1]$ such that $o \sim c_{o} o_{1}+\left(1-c_{o}\right) o_{0}$ (in fact, $c_{o}=\inf \left\{c: c o_{1}+(1-c) o_{0} \succeq o\right\}$ ). Clearly $c_{o_{1}}=1$ and $c_{o_{0}}=0$. By A5, it follows that $o \sim_{\delta} c_{o} O_{1}+\left(1-c_{o}\right) o_{0}$ for all atoms $\delta$. Thus, we must have that $u(\delta, o)=c_{0} u\left(\delta, o_{1}\right)$ for all atoms $\delta$ and all $u \in \mathcal{U}$.

We now construct a state-independent SEU representation using $O$ as the outcome space. Let $S^{\prime}=\operatorname{At}_{\mathcal{T}}\left(T_{0}\right) \times \mathcal{A}_{0} \times E X_{\mathrm{AX}}^{+}(\succeq)$. Define $\pi_{S^{\prime}}^{0}$ by taking $\pi_{S}^{0}(t)=$ $\cup_{\delta \Rightarrow t}\left\{\delta \times A_{0} \times E X_{\mathrm{AX}}^{+}(\succeq)\right\}$, and define $\rho_{S O}(a)\left(\left(\delta, a^{\prime}, \succeq^{\prime}\right)\right)$ to be $a$ if $a \in O ; o_{1}$ if $a \in A_{0}-O$ and $a \succeq_{\delta}^{\prime} a^{\prime}$; and $o_{0}$ otherwise. Let $u^{\prime}$ be defined by taking $u^{\prime}(o)=c_{o}$. Finally, recall that we can take $\mathcal{U}=\left\{u_{\succeq^{\prime}}: \succeq^{\prime} \in E X_{\mathrm{AX}}^{+}(\succeq)\right\}$, where $u_{\succeq^{\prime}}$ represents $\succeq^{\prime}$. Let $p_{\succeq^{\prime}}$ be defined so that $p_{\succeq^{\prime}}\left(\delta, a, \succeq^{\prime \prime}\right)=0$ and, for all $a \in \mathcal{A}_{0}, p_{\succeq^{\prime}}\left(\left\{\left(\delta, a^{\prime}, \succeq^{\prime}\right): a \succeq^{\prime}\right.\right.$ $\left.\left.a^{\prime}\right\}\right)=u_{\succeq^{\prime}}(\delta, a)$. It is easy to check that a probability measure $p_{\succeq^{\prime}}$ can be defined so as to satisfy this constraint. In particular, note that $p_{\succeq^{\prime}}\left(\{\delta\} \times \mathcal{A}_{0} \times\left\{\succeq^{\prime}\right\}\right)=u_{\succeq^{\prime}}\left(\delta, o_{1}\right)$. For all $(\delta, a) \in S$, we have that

$$
u_{\succeq^{\prime}}(\delta, a)=\sum_{a^{\prime}: a \succeq^{\prime} a^{\prime}} p_{\succeq^{\prime}}\left(\delta, a^{\prime}, \succeq^{\prime}\right)=\sum_{a^{\prime} \in \mathcal{A}_{0}} p_{\succeq^{\prime}}\left(\delta, a^{\prime}, \succeq^{\prime}\right) u\left(\rho_{S^{\prime} O}(a)\left(\delta, a^{\prime}, \succeq^{\prime}\right)\right) .
$$

It follows that $\mathcal{P}$ and $u$ represent $\succeq$, where $\mathcal{P}=\left\{p_{\succeq^{\prime}}: \succeq^{\prime} \in E X_{\mathrm{AX}}^{+}(\succeq)\right\}$. As usual, it is straightforward to verify that updating works appropriately.

\section{References}

AHN, D. (2008). Ambiguity without a state space. Review of Economic Studies, 75 (1), 3-28.

- and ERGIN, H. (2007). Framing contingencies, unpublished manuscript.

ALON, S. and LEHRER, E. (2014). Subjective multiple prior probability: A representation of a partial likelihood relation. Journal of Economic Theory, 151, 476-492.

Anscombe, F. J. and Aumann, R. J. (1963). A definition of subjective utility. Annals of Mathematical Statistics, 34, 199-205.

AumANN, R. J. (1962). Utility theory without the completeness axiom. Econometrica, 30, 445-462.

- (1964). Utility theory without the completeness axiom: A correction. Econometrica, 32, 210-212. 
— (1976). Agreeing to disagree. Annals of Statistics, 4 (6), 1236-1239.

BenARTZI, S. and ThaleR, R. H. (2001). Diversification strategies in defined contribution savings plans. American Economic Review, 91 (1), 79-98.

BEWLEY, T. (2002). Knightian decision theory. Decisions in Economics and Finance, 25 (79-110).

Billot, A. and Vergopoulos, V. (2018). Exepcted utility without parsimony. Mathematical Social Sciences, 93, 14-21.

Blume, L., EAsLey, D. and HalPERn, J. (2006). Redoing the foundations of decision theory. In Principles of Knowledge Representation and Reasoning: Proc. Tenth International Conference (KR '06), pp. 14-24.

DekEL, E., LIPMAN, B. and RustichiNI, A. (2001). Representing preferences with a unique subjective state space. Econometrica, 69, 891-934.

Dubra, J., MACChERONI, F. and OK, E. (2004). Expected utility theory without the completeness axiom. Journal of Economic Theory, 115, 118-133.

ELIAZ, K. and OK, E. (2006). Indifference or indecisiveness? choice-theoretic foundations of incomplete preferences. Games and Economic Behavior, 56, 61-86.

FAGIN, R. and HALPERN, J. Y. (1988). Belief, awareness, and limited reasoning. Artificial Intelligence, 34, 39-76.

FeINBERG, Y. (2004). Subjective reasoning-games with unawareness. Tech. Rep. Resarch Paper Series \#1875, Stanford Graduate School of Business.

FischHoff, B., Slovic, P. and Lichtenstein, S. (4). Fault trees: Sensitivity of estimated failure probabilities to problem representation. Journal of Experimental Psychology: Human Perception and Performance, 2, 330-44.

FISHBURN, P. C. (1970). Utility Theory for Decisionmaking. New York: John Wiley and Sons.

- (1987). Reconsiderations in the foundations of decision under uncertainty. The Economic Journal, 97 (388), 825-841.

GalaAbaATAR, T. and KARNI, E. (2013). Subjective expected utility with incomplete preferences. Econometrica, 81, 255-284. 
GhiRARDATO, P. (2001). Coping with ignorance: unforeseen contingencies and nonadditive uncertainty. Economic Theory, 17, 247-276.

GILBOA, I. and SchmeIDLER, D. (2004). Subjective distributions. Theory and Decision, 56, 345-357.

GRABISZEWSKI, K. (2016). On the rejectability of the subjective expected utility theory. BE Journal of Theoretical Economics, 16, 437-454.

HALPERN, J. Y. (2001). Alternative semantics for unawareness. Games and Economic Behavior, 37, 321-339.

- and RÊGO, L. C. (2006). Extensive games with possibly unaware players. In Proc. Fifth International Joint Conference on Autonomous Agents and Multiagent Systems, pp. 744-751, full version available at arxiv.org/abs/0704.2014.

Heifetz, A., Meier, M. and Schipper, B. (2006). Interactive unawareness. Journal of Economic Theory, 130, 78-94.

Johnson, E., Hershey, J., Meszaros, J. and Kunreuther, H. (1993). Framing, probability distortions, and insurance decisions. Journal of Risk and Uncertainty, 7, 35-51.

KARNI, E. (2006). Subjective expected utility theory without states of the world. Journal of Mathematical Economics, 42, 325-342.

— and VIERØ, M.-L. (2013). "Reverse Bayesianism": A choice-based theory of growing awareness. American Economic Review, 103 (7), 2790-2810.

Krantz, D. H., Luce, R. D., Suppes, P. and TVersky, A. (1971). Foundations of Measurement, Vol 1: Additive and Polynomial Representations. New York: Academic Press.

KREPS, D. (1992). Static choice and unforeseen contingencies. In P. Dasgupta, D. Gale and O. Hart (eds.), Economic Analysis of Markets and Games: Essays in Honor of Frank Hahn, Cambridge, Mass.: MIT Press.

KREPS, D. M. (1979). A representation theorem for "preference for flexibility". Econometrica, 47 (3), 565-77.

- (1988). Notes on the Theory of Choice. Boulder, Colo.: Westview Press. 
LIPMAN, B. (1999). Decision theory without logical omniscience: Toward an axiomatic framework for bounded rationality. Review of Economic Studies, 66, 339361.

LUCE, R. D. (1990). Rational versus plausible accounting equivalences in preference judgments. Psychological Science, 1, 225-234, reprinted with minor changes in Ward Edwards (Ed.), Utility Theories: Measurements and Applications, pp. 187206. Boston: Kluwer, 1992.

MACHINA, M. (2006). States of the world and state of decision theory. In D. Meyer (ed.), The Economics of Risk, Kalamazoo, MI: W. E. Upjohn Institute.

MANDLER, M. (2001). A difficult choice in preference theory: rationality implies completeness or transitivity but not both. In E. Millgram (ed.), Varieties of Practical Reasoning, Cambridge MA: MIT Press.

McNeil, B. J., Pauker, S. J., Sox JR., H. C. and TVersky, A. (1982). On the elicitation of preferences for alternative therapies. New England Journal of Medicine, 306, 1259-1262.

ModicA, S. and RUSTICHINI, A. (1999). Unawareness and partitional information structures. Games and Economic Behavior, 27 (2), 265-298.

NAU, R. (2006). The shpae of incomplete preferences. Annals of Statistics, 34, 24302448.

RANTALA, V. (1982). Impossible worlds semantics and logical omniscience. Acta Philosophica Fennica, 35, 18-24.

Rockafellar, R. T. (1970). Convex Analysis. Princeton, N.J.: Princeton University Press.

SAVAGE, L. J. (1954). Foundations of Statistics. New York: Wiley.

SCHMEIDLER, D. (1989). Subjective probability and expected utility without additivity. Econometrica, 57, 571-587.

ScotT, D. (1964). Measurement structures and linear inequalities. Journal of Mathematical Psychology, 1, 233-247.

ThaleR, R. H. and SUNSTEIN, C. (2009). Nudge: Improving Decisions About Health, Wealth, and Happiness. Penguin Books. 
TVersky, A. and KoehleR, D. J. (1994). Support theory: A nonextentional representation of subjective probability. Psychological Review, 101 (4), 547-67. 